\title{
Transcriptomic Profiling Discloses Molecular and Cellular Events Related to Neuronal Differentiation in SH-SY5Y Neuroblastoma Cells
}

\section{Pezzini, Francesco}

2017-05

Pezzini , F , Bettinetti , L , Di Leva , F , Bianchi , M , Zoratti , E , Carrozzo , R , Santorelli , F M , Delledonne , M , Lalowski , M \& Simonati , A 2017 , ' Transcriptomic Profiling Discloses Molecular and Cellular Events Related to Neuronal Differentiation in SH-SY5Y

Neuroblastoma Cells ' , Cellular and Molecular Neurobiology , vol. 37 , no. 4 , pp. 665-682 . https://doi.org/10.1007/s

http://hdl.handle.net/10138/236792

https://doi.org/10.1007/s10571-016-0403-y

publishedVersion

Downloaded from Helda, University of Helsinki institutional repository.

This is an electronic reprint of the original article.

This reprint may differ from the original in pagination and typographic detail.

Please cite the original version. 


\title{
Transcriptomic Profiling Discloses Molecular and Cellular Events Related to Neuronal Differentiation in SH-SY5Y Neuroblastoma Cells
}

\author{
Francesco Pezzini ${ }^{1}$ - Laura Bettinetti ${ }^{2}$ - Francesca Di Leva ${ }^{2}$ - Marzia Bianchi ${ }^{3}$. \\ Elisa Zoratti ${ }^{4,7} \cdot$ Rosalba Carrozzo $^{3} \cdot$ Filippo M. Santorelli $^{5} \cdot$ Massimo Delledonne $^{2}$. \\ Maciej Lalowski ${ }^{6} \cdot$ Alessandro Simonati $^{1}$ (D)
}

Received: 21 March 2016/ Accepted: 9 June 2016/Published online: 15 July 2016

(c) Springer Science+Business Media New York 2016

\begin{abstract}
Human SH-SY5Y neuroblastoma cells are widely utilized in in vitro studies to dissect out pathogenetic mechanisms of neurodegenerative disorders. These cells are considered as neuronal precursors and differentiate into more mature neuronal phenotypes under selected growth conditions. In this study, in order to decipher the pathways and cellular processes underlying neuroblastoma cell differentiation in vitro, we performed systematic transcriptomic (RNA-seq) and bioinformatic analysis of SH-SY5Y cells differentiated according to a two-step
\end{abstract}

Electronic supplementary material The online version of this article (doi:10.1007/s10571-016-0403-y) contains supplementary material, which is available to authorized users.

Maciej Lalowski

maciej.lalowski@helsinki.fi

$\triangle$ Alessandro Simonati

alessandro.simonati@univr.it

1 Department of Neuroscience, Biomedicine and Movement, University of Verona, Verona, Italy

2 Department of Biotechnologies, University of Verona, Verona, Italy

3 Unit for Neuromuscular and Neurodegenerative Disorders, Laboratory of Molecular Medicine, IRCCS Bambino Gesù Children's Hospital, Rome, Italy

4 Applied Research on Cancer-Network (ARC-NET), University of Verona, Verona, Italy

5 Unit for Neuromuscular and Neurodegenerative Disorders, Laboratory of Molecular Medicine, IRCCS Stella Maris, Calambrone-Pisa, Italy

6 Medicum, Biochemistry/Developmental Biology Meilahti Clinical Proteomics Core Facility, University of Helsinki, Helsinki, Finland

7 Present Address: Aptuit s.r.l., Verona, Italy paradigm: retinoic acid treatment followed by enriched neurobasal medium. Categorization of 1989 differentially expressed genes (DEGs) identified in differentiated cells functionally linked them to changes in cell morphology including remodelling of plasma membrane and cytoskeleton, and neuritogenesis. Seventy-three DEGs were assigned to axonal guidance signalling pathway, and the expression of selected gene products such as neurotrophin receptors, the functionally related SLITRK6, and semaphorins, was validated by immunoblotting. Along with these findings, the differentiated cells exhibited an ability to elongate longer axonal process as assessed by the neuronal cytoskeletal markers biochemical characterization and morphometric evaluation. Recognition of molecular events occurring in differentiated SH-SY5Y cells is critical to accurately interpret the cellular responses to specific stimuli in studies on disease pathogenesis.

Keywords SH-SY5Y differentiation - RNA-seq analysis · Axonal guidance signalling $\cdot$ Semaphorins $\cdot$ Neuronal markers $\cdot$ Axonal elongation

$\begin{array}{ll}\text { Abbreviations } \\ \text { BDNF } & \text { Brain-derived neurotrophic factor } \\ \text { db-cAMP } & \text { Dibutyryl-cyclic AMP } \\ \text { DEG } & \text { Differentially expressed gene } \\ \text { FDZs } & \text { Frizzled receptors } \\ \text { FPKM } & \text { Fragments per kilobase per million mapped } \\ & \text { reads } \\ \text { IPA } & \text { QIAGEN's ingenuity }{ }^{\circledR} \text { pathway analysis } \\ \log _{2} \mathrm{FC} & \text { Log }_{2} \text { fold change (ratio between averaged } \\ & \begin{array}{l}\text { FPKM of RA-NBM differentiated cells and } \\ \text { the averaged FPKM of untreated cells) }\end{array} \\ \text { NGF } & \text { Nerve growth factor } \\ \text { NGFR } & \text { Nerve growth factor receptor }\end{array}$




\section{NRP1 Neuropilin-1}

NTF4 Neurotrophin-4

NTRK2 Neurotrophic tyrosine kinase receptor type 2

PLXNA4 Plexin A4

pNF-H Phosphorylated heavy-chain neurofilament proteins

pNF-M Phosphorylated medium-chain neurofilament proteins

RA All trans retinoic acid

RA-NBM 9-day-long differentiation paradigm in which SH-SY5Y cells were pre-differentiated in RA medium (6 days) and subsequently treated with neurobasal medium enriched with neurotrophic factors (3 days)

RA-BDNF 9-day-long differentiation paradigm in which SH-SY5Y cells were pre-differentiated in RA medium (6 days) and subsequently treated with serum-free medium containing rhBDNF (3 days)

SEMA Semaphorin

SLITRK6 SLIT and NTRK-like family

SYN1 Synapsin I

\section{Introduction}

SH-SY5Y is a well-established cell line which was subcloned from the SK-N-SH clone, originally obtained by a bone marrow biopsy of a 4-year-old female patient affected by neuroblastoma (Biedler et al. 1978). Neuroblastoma cells are morphologically heterogeneous and at least three different phenotypes can be recognized in vitro: small, round-shaped neuroblastic cells with short neurites (N-type), substrate-adherent cells (S-type) characterized by a flat morphology, and I-type cells, which display an intermediated phenotype between $\mathrm{N}$ - and S-type (Walton et al. 2004). Each subgroup could be further identified by the differential expression of cytoskeletal markers, such as nestin, $\beta$-III tubulin, neurofilaments, vimentin and GFAP (Ross and Spengler 2007). SH-SY5Y cell line is mainly characterized by an $\mathrm{N}$-phenotype, although adherent cells are also observed (Bell et al. 2013; Ross et al. 1983).

SH-SY5Y cells are considered to be neuronal precursors and several growth conditions were reported to switch these cells to more mature "neuronal-like" phenotype (for a comprehensive review see Kovalevich and Langford 2013). All trans retinoic acid (RA), a vitamin A metabolite involved in growth and development, is known to induce neuronal differentiation of SH-SY5Y cells as well as to inhibit the cellular proliferation (Borriello et al. 2006;
Cheung et al. 2009; Constantinescu et al. 2007; Cuende et al. 2008; Nakamura et al. 2003; Pahlman et al. 1984). A more mature neuronal phenotype can be reached using growth medium supplemented with specific chemical factors, such as recombinant human brain-derived neurotrophic factor (BDNF), nerve growth factor (NGF), dibutyryl-cyclic AMP (db-cAMP), phorbol esters and others (Agholme et al. 2010; Dwane et al. 2013; Encinas et al. 2000; Gimenez-Cassina et al. 2006; Jamsa et al. 2004; Kou et al. 2008; Kume et al. 2008; Sarkanen et al. 2007). Thereafter, SH-SY5Y cells have been largely utilized to investigate the disease mechanisms associated with various neurodegenerative disorders (Agholme et al. 2014; Ferreira et al. 2013; Hadzhieva et al. 2013; Jamsa et al. 2004; Ke et al. 2012; Krishna et al. 2014; Lim et al. 2015; Lopes et al. 2010, 2012; Palomo et al. 2011; Scifo et al. 2013, 2015).

The molecular events, which underlie differentiation of SH-SY5Y have not been fully characterized. It is conceivable that variations occur in the expression of sets of genes, which exert their effects in different compartments of the cell according to sequentially orchestrated movements. Recently, the functional networks and biological functions involved in SH-SY5Y differentiation were analysed by gene expression techniques (microarray and quantitative real-time PCR) focusing on both genes and miRNA expression profiles of SH-SY5Y which were differentiated using RA alone or in combination with BDNF (Batagov et al. 2013; de Bittencourt Pasquali et al. 2016; Goldie et al. 2014; Korecka et al. 2013).

The major aim of this study was to identify putative changes in expression of genes in differentiated $\mathrm{SH}$ SY5Y cells, grown in RA and neurobasal medium enriched with neurotrophic factors (modified from Gimenez-Cassina et al. 2006, and hereafter referred as RA-NBM treatment), and to evaluate their differentiation towards a neuronal phenotype. To reach our goal we scrutinized the molecular mechanisms underlying differentiation by whole transcriptome profiling (RNA-seq), a powerful technology which allows studying the expression of thousands of genes simultaneously (Wang et al. 2009). Identified, differentially expressed genes (DEGs) were subsequently examined using bioinformatic tools, to recognize biological functions and molecular pathways relevant for the differentiation process. Furthermore, we examined the differential expression of a subset of proteins encoded by DEGs and selected neuronal cytoskeletal markers using morphological and biochemical tools. Finally, the neuronal "maturation state" of differentiated, SH-SY5Y cells was assessed in morphometric analyses to evaluate the growth features of axonal processes and their branches. 


\section{Materials and Methods}

\section{Cell Cultures and Differentiation Media}

Human neuroblastoma SH-SY5Y cells (catalogue number \#94030304, purchased by European Collection of Cell Cultures) were cultured in DMEM-High glucose medium (DMEM/HIGH) supplemented with $15 \%$ foetal bovine serum (FBS), $2 \mathrm{mM}$ L-glutamine and $1 \%$ non-essential amino acids (all purchased from Euroclone, Pero, Italy) at $37{ }^{\circ} \mathrm{C}$ and $5 \% \mathrm{CO}_{2}$. For neuronal differentiation, we set up a 9-day-long paradigm (RA-NBM treatment) including a 6-day pre-differentiation step in DMEM/HIGH supplemented with $5 \%$ FBS and $10 \mu \mathrm{M}$ RA (Sigma-Aldrich, Milan, Italy) followed by a 3-day differentiation step in neurobasal medium (GIBCO, Life Technologies) enriched with $50 \mathrm{ng} \mathrm{ml}^{-1}$ recombinant human BDNF (rhBDNF, Peprotech, Rocky Hill, NJ, USA), $2 \mathrm{mM}$ db-cAMP (Sigma-Aldrich), $20 \mathrm{mM} \mathrm{KCl}, \mathrm{B} 27$ supplement and $1 \%$ GlutaMAX (both purchased form GIBCO, Life Technologies, Monza, Italy). Control (not treated, NT) cells were grown under basal conditions at low FBS concentration (5\%) for comparison with RA-NBM treated cells. In another set of experiments, the cells were differentiated in RA for 6 days followed by 3 days of serum-free medium containing $50 \mathrm{ng} \mathrm{ml}^{-1}$ rhBDNF (RA-BDNF medium). This paradigm was used to assess the expression of neuronal cytoskeletal markers on Western blots, and to perform flow cytometry assays for cell death investigation. Cells $\left(8 \times 10^{3}\right.$ per $\left.\mathrm{cm}^{2}\right)$ were seeded in T75 flasks or on coverslips previously coated with ECMax gel (SigmaAldrich), allowed to adhere for $24-48 \mathrm{~h}$ and exposed to basal or differentiation media. Media were routinely changed every 2-3 days and the morphological features of the cultures were checked by phase contrast microscopy. For quantitative evaluation, the cells grown under basal conditions were harvested after 6 days to avoid the overgrowth of the culture. Growth paradigms are summarized in Fig. S1 of Electronic Supplementary Material 1 (ESM1).

\section{RNA-seq and Transcriptomic Analysis}

Whole transcriptome analysis was performed by RNA-seq technology on untreated and RA-NBM differentiated cells. Cells were collected in three independent experiments and total RNA was isolated using TRI Reagent. RNA purity and quantity was checked using Nanodrop 1000 (Agilent Technologies, Santa Clara, CA, USA), while RNA integrity (RNA integrity number $\geq 8.0$ ) was assessed using RNA 6000 Nano Kit (Agilent). Indexed cDNA libraries were prepared using TruSeq RNA Sample Prep Kit v2 (Illumina, San Diego, CA, USA) according to manufacturer's instructions. Adapter-ligated cDNA libraries with an insert size of 200-250 bp were size selected on $1.5 \%$ agarose gel cassettes using Pippin Prep instrument (Sage Science, Beverly, MA, USA). Quality of libraries was checked using high-sensitivity DNA Kit (Agilent). Libraries were quantified by real-time PCR against a standard curve with KAPA Library Quantification Kit (KapaBiosystems, Wilmington, MA, USA). Libraries were then pooled at equimolar concentration and sequenced using Illumina HiSeq 1000 sequencer with TruSeq SBS Kit v3-HS and TruSeq PE Cluster kit v3-HS (Illumina), by applying standard manufacturer protocols. About 30 million pairedend $(100 \mathrm{bp} \times 2)$ reads were generated for each sample (6 Gbps). Quality of reads obtained from each sample was assessed using FastQC software (http://www.bioinfor matics.babraham.ac.uk/projects/fastqc/), and reads with more than $10 \%$ of undetermined bases or more than 50 bases with a quality score $<7$ were discarded. Reads were then clipped from adapter sequences using Scythe software (https://github.com/vsbuffalo/scythe), and low-quality ends (Q score $<20$ on a 10-nt window) were trimmed with Sickle (https://github.com/vsbuffalo/sickle). Alignment of reads to reference human genome (hg19) was performed using TopHat 2 (http://tophat.cbcb.umd.edu/) and quality checked with RSeQC software (http://rseqc.sourceforge. net/). In order to facilitate a comparison of expression profiles, the normalized expression values expressed as fragments per kilobase per million mapped reads (FPKM) for each transcript were calculated. The differential expression of each gene was presented as a $\log _{2}$ fold change $\left(\log _{2} \mathrm{FC}\right)$, by determining the ratio between averaged FPKM of RA-NBM differentiated cells and the averaged FPKM of untreated cells. Transcripts showing a $\left|\log _{2}(\mathrm{FC})\right| \geq 1$ and a false discovery rate (FDR, $q$-value) $\leq 0.05$ were assigned as differentially expressed.

\section{Bioinformatic Analysis of Transcriptomic Data}

Differentially expressed genes (hereafter called as DEGs, corresponding to identified transcripts) were evaluated by QIAGEN's Ingenuity ${ }^{\circledR}$ Pathway Analysis (IPA ${ }^{\circledR}$, Spring release 2015, QIAGEN Redwood City, USA, www.qiagen. com/ingenuity), to recognize meaningful biological processes and molecular pathways. Specifically, we performed a downstream effects analysis, focusing mainly on the IPA categories of molecular and cellular functions and physiological system development and function; canonical pathways were analysed as well. For each biological annotation, IPA algorithm calculated a Benjamini-Hochberg multiple testing correction $p$ value (B-H $p$ value), which depicts robustness of correlation between a subset of DEGs in our dataset with a given biological function (or total number of assigned genes to a pathway). Moreover, 
the IPA algorithm allowed predicting the activation or the inhibition state of a given biological function by assigning the related " $z$-score". Only most meaningful functional annotations, showing the lowest $p$ value were evaluated; in addition, only -scores $\geq+2$ (predicted activation) or $\leq-2$ (predicted inhibition) were considered. For canonical pathway analysis, the IPA algorithm calculates the percentage of overlap, which depicts a percentage of assigned DEGs in comparison to the total number of molecules constituting the pathway. Finally, several differentially expressed genes, which were assigned to the most significant biological functions and canonical pathways, were selected to be further investigated at the protein level by semi-quantitative Western blotting.

\section{Quantitative Real-Time PCR (qRT-PCR)}

The expression of Plexin A4 transcript (PLXNA4) was assessed by qRT-PCR in RNA samples isolated from untreated and RA-NBM differentiated cells in four independent experiments. In brief, $1.5 \mu \mathrm{g}$ of RNA was treated with DNase I Amplification Grade (Invitrogen, Life Technologies) before the reverse transcription to avoid genomic DNA contamination. The reverse transcription was performed on $200 \mathrm{ng}$ DNase-treated RNA by GeneAmp RNA PCR Core Kit (Applied Biosystem, Life Technologies). Subsequently, cDNAs were amplified in ABI 7300 Fast real-time PCR (Applied Biosystem) using iTaq Universal SYBR Green Supermix (Bio-Rad Laboratories S.r.l, Segrate, Milan, Italy). Primers used in this study were purchased from Bio-rad Laboratories (PrimePCR $^{\mathrm{TM}} \mathrm{SYBR}^{\circledR}$ Green Assay PLXNA4, human, unique assay ID: qHsaCID0018081; PrimePCR ${ }^{\mathrm{TM}}$ Assay GAPDH, unique assay ID: qHsaCED0038674). For the absolute quantification, two independent standard curves were created using PrimePCR ${ }^{\mathrm{TM}}$ Template for $\mathrm{SYBR}^{\circledR}$ Green Assay, specific for each gene (Bio-Rad). Following interpolation of $\mathrm{Ct}$ values with the relative standard curve, the PLNXA4/GAPDH ratio was calculated and normalized to the expression values of undifferentiated cells. Data were reported as mean \pm s.e.m. and the statistical analysis was performed using two-tailed Student's $t$ test.

\section{Western Blotting Analysis}

The SH-SY5Y cells were collected at specific time points (also referred as DIV, days in vitro), rinsed twice with PBS and then homogenized in RIPA buffer containing inhibitors of proteases (Roche Diagnostics $\mathrm{GmbH}$, Milan, Italy) at $4{ }^{\circ} \mathrm{C}$, for $1 \mathrm{~h}$. Cell lysates were centrifuged at $16,000 \mathrm{~g}$ for $15 \mathrm{~min}$ and the supernatants were collected and stored at $-80{ }^{\circ} \mathrm{C}$. Protein content was determined using the Pierce BCA protein assay kit (Thermo Fisher Scientific, Monza,
Italy). For Western blotting, $20 \mu \mathrm{g}$ of protein lysates were resolved on SDS-PAGE under denaturing conditions and then transferred onto $0.22 \mu \mathrm{m}$ PVDF membranes (Bio$\mathrm{Rad})$. The membranes were incubated for $1 \mathrm{~h}$ in blocking solution (TBS/0.1\% Tween20 with $10 \%$ non-fat dry milk). Incubation with primary antibodies (see Online Resource ESM 2) was performed overnight at $4{ }^{\circ} \mathrm{C}$, followed by incubation for $1 \mathrm{~h}$ at RT with species-specific HRP-conjugated antibodies (ECL Mouse and Rabbit IgG HRP linked $f(a b ') 2$ fragment, 1:10,000, Amersham, GE Healthcare Europe $\mathrm{GmbH}$, Milan, Italy; Peroxidase AffiniPure Donkey Anti-Sheep IgG $(\mathrm{H}+\mathrm{L}), 1: 10,000$, Jackson ImmunoResearch, Suffolk, UK). Chemiluminescent detection was performed with Immobilon Western Chemiluminescent HRP Substrate (Merck Millipore S.p.a., Vimodrone, Milan, Italy) according to manufacturer's instructions. For quantitative evaluation, the cells were collected from at least four independent experiments and lysates were assessed in the same electrophoretic run. Data were assessed using ImageJ software (Schneider et al. 2012) and normalized to a loading control (either actin or GAPDH). Data were reported as mean \pm s.e.m. The statistical analysis was performed using two-tailed Student's $t$ test, considering as significant a $p$ value $\leq 0.05$ and as highly significant a $p$ value $\leq 0.01$.

\section{Immunofluorescence Assay}

The cells were fixed in $4 \%$ paraformaldehyde (PFA) for $20 \mathrm{~min}$ at RT or in absolute methanol at $-20{ }^{\circ} \mathrm{C}$ for $10 \mathrm{~min}$. Coverslips were rinsed twice in PBS and incubated in the blocking solution (10\% normal goat serum, NGS in PBS) for $1 \mathrm{~h}$ at RT. For PFA fixed cells, PBS/0.3\% Triton solution was used to permeabilize the membranes. Cells were exposed to primary antibodies in an incubation buffer (PBS with $1 \%$ NGS) overnight at $4{ }^{\circ} \mathrm{C}$ (Online Resource ESM 2). Anti-mouse and anti-rabbit secondary antibodies conjugated with AlexaFluor 488 or AlexaFluor 594 dyes (1:800; Molecular Probes, Life Technologies) were used for $1 \mathrm{~h}$ at RT. The nuclei were counterstained with $5 \mu \mathrm{g} / \mathrm{ml}$ DAPI (4',6-diamidino-2-phenylindole di-hydrochloride; Sigma-Aldrich). Labelled cells were analysed under Axiolab microscope (Carl Zeiss, Milan, Italy) equipped with HBO 50 mercury vapour short-arc lamp for incident lightfluorescence. Unlabelled cells were examined by brightfield microscopy. Images were acquired on AxioCamera using AxioVision 4.3 software (Carl Zeiss).

\section{Morphometric Evaluation}

Differentiated and undifferentiated SH-SY5Y cells were assessed in four independent experiments. Images were acquired at $10 \times$ magnitude and at least 1500 cells per cell 
line were evaluated. Both number and length of all neurofilament-immunolabelled structures were calculated. The elongation of neuronal processes was investigated by immunofluorescence using SMI-31R antibody (Covance, Rome, Italy), which specifically reacts with a phosphorylated epitope of a heavy chain of neurofilament (pNF-H) and, to a lesser extent, with the medium chain (pNF-M; Online Resource ESM 2). NeuronJ plugin for ImageJ software (Meijering 2010) was used to manually trace neurofilament-positive structures after twofold increase of the acquired pictures. Primary processes, sprouting from the cell body were traced along with the secondary processes (branching from a primary process) and, if present, with tertiary processes (branching from secondary structures). Moreover, only primary processes longer than $30 \mu \mathrm{m}$ were considered. DAPI-positive nuclei were automatically quantified by CellProfiler 2.1.1 (Kamentsky et al. 2011) and used to normalize the number of processes on the cellular density. Data were collected from at least four independent experiments and reported as mean \pm s.e.m. The results were evaluated statistically using two-tailed Student's $t$ test, considering as significant a $p$ value $\leq 0.05$ and as highly significant a $p$ value $\leq 0.01$.

\section{Data Availability}

RNA-seq data discussed in this publication have been deposited in NCBI's Gene Expression Omnibus and are accessible through GEO Series accession number GSE77383 (https://www.ncbi.nlm.nih.gov/geo/query/acc. cgi?acc $=$ GSE77383).

\section{Results}

\section{Transcriptomic Profile of Differentiated SH-SY5Y}

We compared the whole transcriptome expression profiles of RA-NBM differentiated cells versus those which were non-differentiated and identified 1989 DEGs, of which 1958 (namely, 1226 up- and 732 down-regulated) were further scrutinized (Fig. 1a and Online Resource ESM 3). Using downstream effects analysis of IPA algorithm, we focused on two major categories of biological functions (Fig. 1b and Online Resource ESM 4). Within the molecular and cellular functions category, the sub-categories related to changes in cell growth rate and morphology, as well as to rearrangements of cytoskeleton and plasma membrane processes were of major interest. We subsequently scrutinized two categories, cell morphology (B-H $p$ value range $=1.55 \mathrm{E}-37$ to $8.47 \mathrm{E}-08,559$ DEGs) and Cellular Assembly and Organization (B-H $p$ value range $=1.86 \mathrm{E}-27$ to $8.47 \mathrm{E}-08,470$ DEGs, Fig. 1b). Confined annotations included remodelling processes of plasma membrane and cytoskeleton, such as formation of cellular protrusions $(\mathrm{B}-\mathrm{H} p$ value $=2.98 \mathrm{E}-14,196$ DEGs, $\mathrm{z}$-score $=3.408$ ), outgrowth of plasma membrane projections, formation of plasma membrane projections and organization of cytoskeleton (all with statistically significant $z$-scores $>+2$; Fig. 1c and Online Resource ESM 5A). Other statistically meaningful associations were linked to early steps of neuronal differentiation, i.e., neuritogenesis $(\mathrm{B}-\mathrm{H} p$ value $=1.56 \mathrm{E}-11,132 \mathrm{DEGs}, z$-score $=2.602)$ and outgrowth of neurites. The $z$-scores $>+2$ calculated by IPA algorithm suggested that all these annotations (and related cellular functions) were likely increased in this experimental setting. These annotations were shared with nervous system development and function, which was one of the most enriched categories of physiological system development and function class (B-H $p$ value $1.69 \mathrm{E}-23$ to 1.24E-07, 464 DEGs). In this category we found other cellular functions strongly associated with neuronal biology, such as proliferation of neuronal cells (B-H $p$ value $=1.11 \mathrm{E}-14,141$ DEGs, $z$-score $=2.587$ ), outgrowth of neurons $(z$-score $=2.721)$ and quantity of neurons $(z$-score $=2.410)$, all predicted to be activated (Fig. 1c and Online Resource ESM 5B). Other noteworthy annotations related to differentiation process, namely differentiation of cells (483 DEGs, $z$-score $=3.863$ ) and differentiation of nervous system (109 DEGs, $z$ score $=3.380$ ) were assigned to Cellular Development category (Online Resource ESM 6A). In the same category proliferation of tumour cells lines displayed a negative $z$ score $(z$-score $=-2.242)$. Cell Death and Survival category $(\mathrm{B}-\mathrm{H} p$ value $=1.24 \mathrm{E}-33$ to $1.18 \mathrm{E}-07,697 \mathrm{DEGs}$ ) encompassed many functions, which predicted to be either increased (linked to apoptotic processes, i.e., apoptosis of blood cells, $z$-score $=2.262$ ) or decreased (cell death of neuroglia, $z$-score $=-1.537$; see Online Resource ESM $6 \mathrm{D})$.

\section{Axonal Guidance Signalling Pathway}

We further queried the association of DEGs with canonical pathways and interestingly identified axonal guidance signalling as one of the most highly over-represented pathways $(-\log \mathrm{B}-\mathrm{H} p$ value $=4.4,73$ DEGs enclosed, $16.9 \%$ overlap; Fig. 2a). DEGs involved in this pathway encoded for extracellular growth factors, cell surface receptors or intra-cytoplasmic adapter proteins, which jointly converged to downstream functions related to cytoskeletal and axonal dynamics (most of these DEGs were up-regulated, see Online Resource ESM 7). Specifically, 8 and 3 genes encoded for different members of semaphorins (SEMA) family and related membrane receptors (NRP1, PLXNA2 and $P L X N D 1$ ), respectively. Together, these molecules 
A

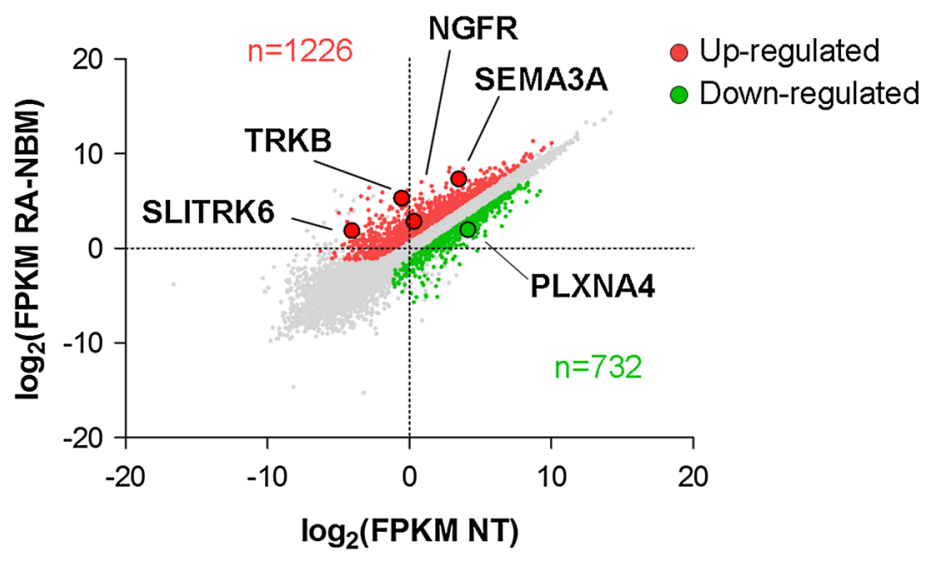

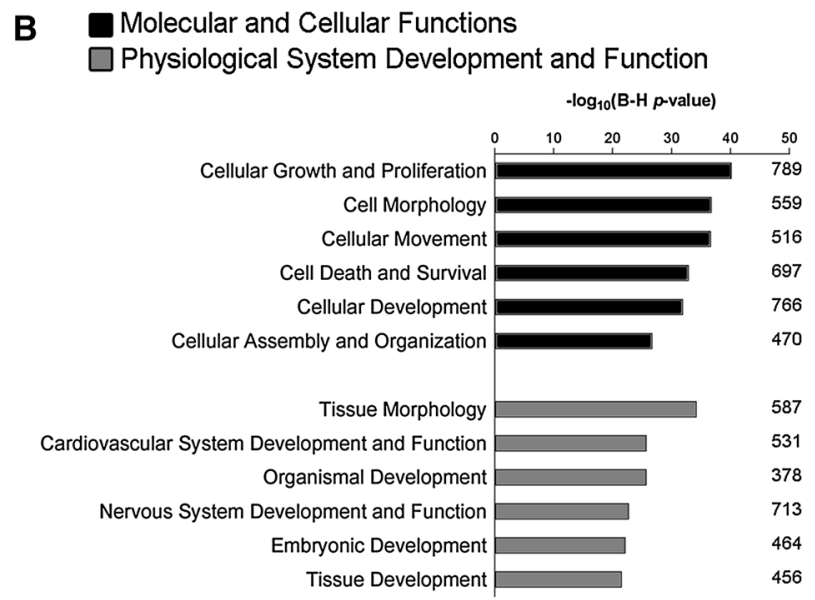

Fig. 1 Comparative analysis of RA-NBM differentiated and nondifferentiated cells. a Scatter plot representation of transcript expression levels (reported as $\log _{2}$ (FPKM) of differentiated, RA$\mathrm{NBM}$, on $y$ axis as compared to non-differentiated cells, NT on $x$ axis). Coloured dots represent differentially expressed transcripts (thresholds: $\mid \log _{2} \mathrm{FCl} \geq 1$ and $q$-value $\leq 0.05$ ), which were either upregulated $(n=1226$, red dots) or down-regulated $(n=732$, green dots). DEGs with $\log _{2} \mathrm{FC}$ values equal to $+\mathrm{INF}$ (infinity) or - INF are not presented in the figure, but were analysed in functional bioinformatic queries. The differentially expressed transcripts, which were further investigated on the protein level, are indicated. b Survey of downstream effects linked to differentially expressed genes. Bars represent the range of $-\log (\mathrm{B}-\mathrm{H} p$ values $)$, calculated by the Benjamini-Hochberg multiple testing correction procedure, for each IPA category assigned to molecular and cellular functions and physiological system development and function classes (upper axis). The number of genes enclosed in each category is reported. Similarly, as in the molecular and cellular function IPA class analysis, the most significant categories were related to changes in cellular morphology, proliferation and development. Nervous system development and

influence and finely regulate remodelling processes of cellular cytoskeletal structures (actin filament reorganization) and axons (axon outgrowth, growth cone attraction/
C
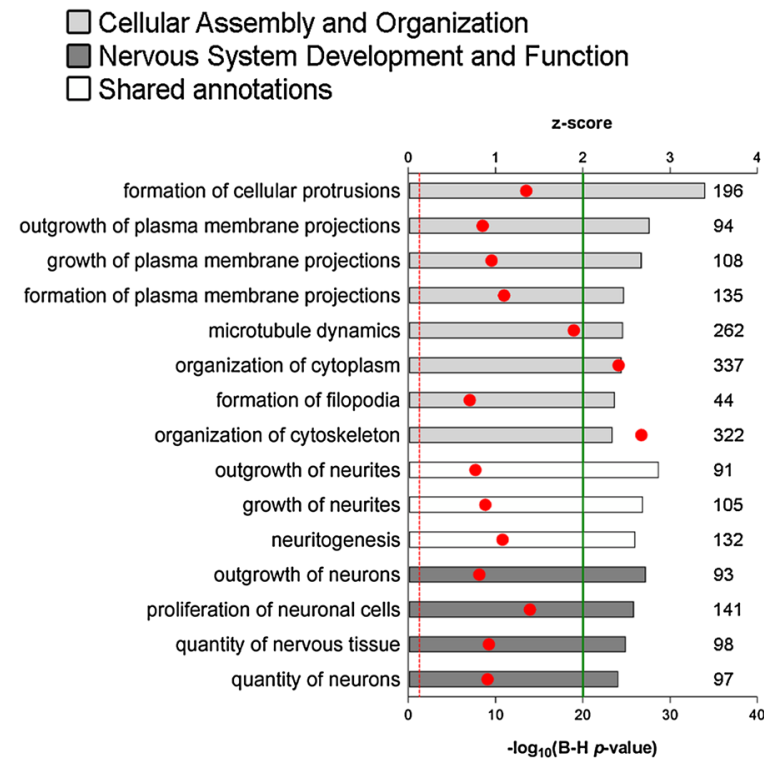

function signified one of the most over-represented categories (464 genes enclosed) among the physiological system development and function group, suggestive for neuronal involvement. c Cellular assembly and organization and nervous system development and function categories were mined to link associated biological functions. Bars represent $z$-scores calculated by IPA algorithm (upper axis), only annotations showing a significant $z$-score $\geq+2$ (marked by green line) were considered. B-H $p$ values (red dots, lower axis), calculated by the Benjamini-Hochberg multiple testing correction procedure indicate significance of associations between a subset of DEGs in our dataset and a specific biological function; a threshold of 1.3 (red line) represents a $p$ value $\leq 0.05$. The number of DEGs fitting into a specific biological function is presented; shared functions are shown in white. Most significantly enriched annotations were denoted as involvement in the re-arrangements of a cellular cytoskeleton, elongation of cellular processes and more specifically in the growth of neuronal structures. Other functions, namely outgrowth of neurons, proliferation of neuronal cells and quantity of neurons represented cues for neuronal commitment. For a complete list of annotations see Online Resources ESM 5 and ESM 6 (Color figure online)

repulsion and axon turning response; Fig. 2b). Moreover, other up-regulated DEGs encoded for BDNF, NTF4 (neurotrophin-4) and their membrane receptor TrkB (also 
A

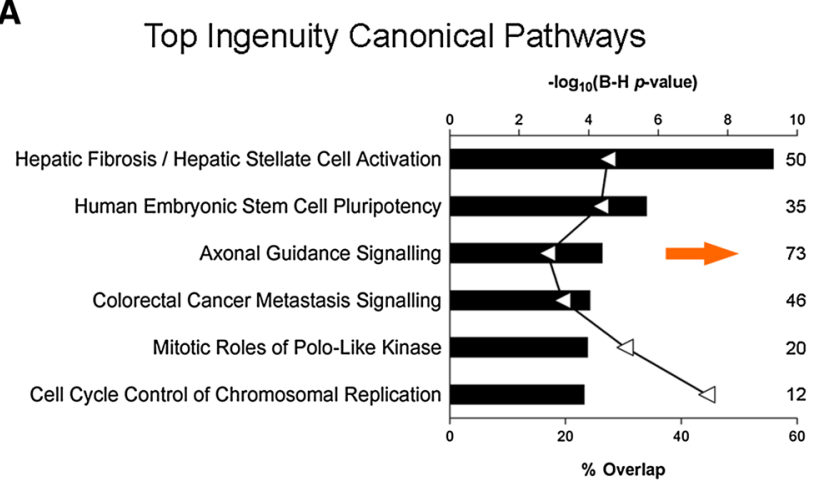

C

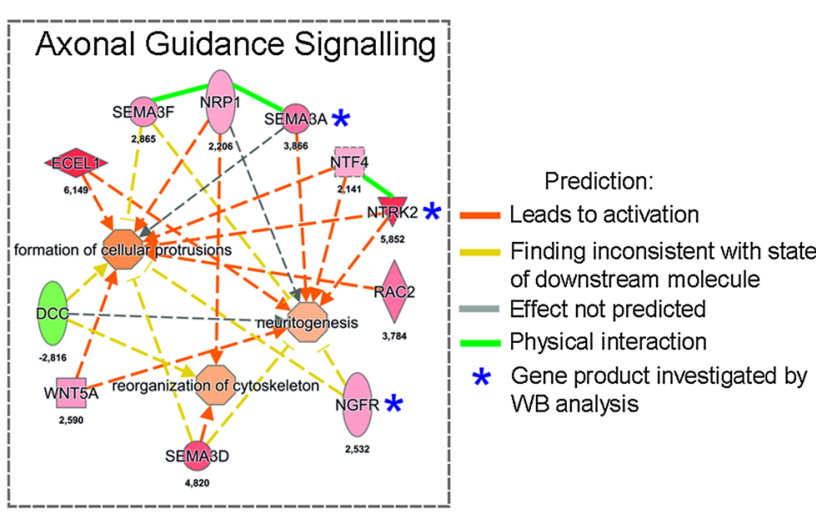

B

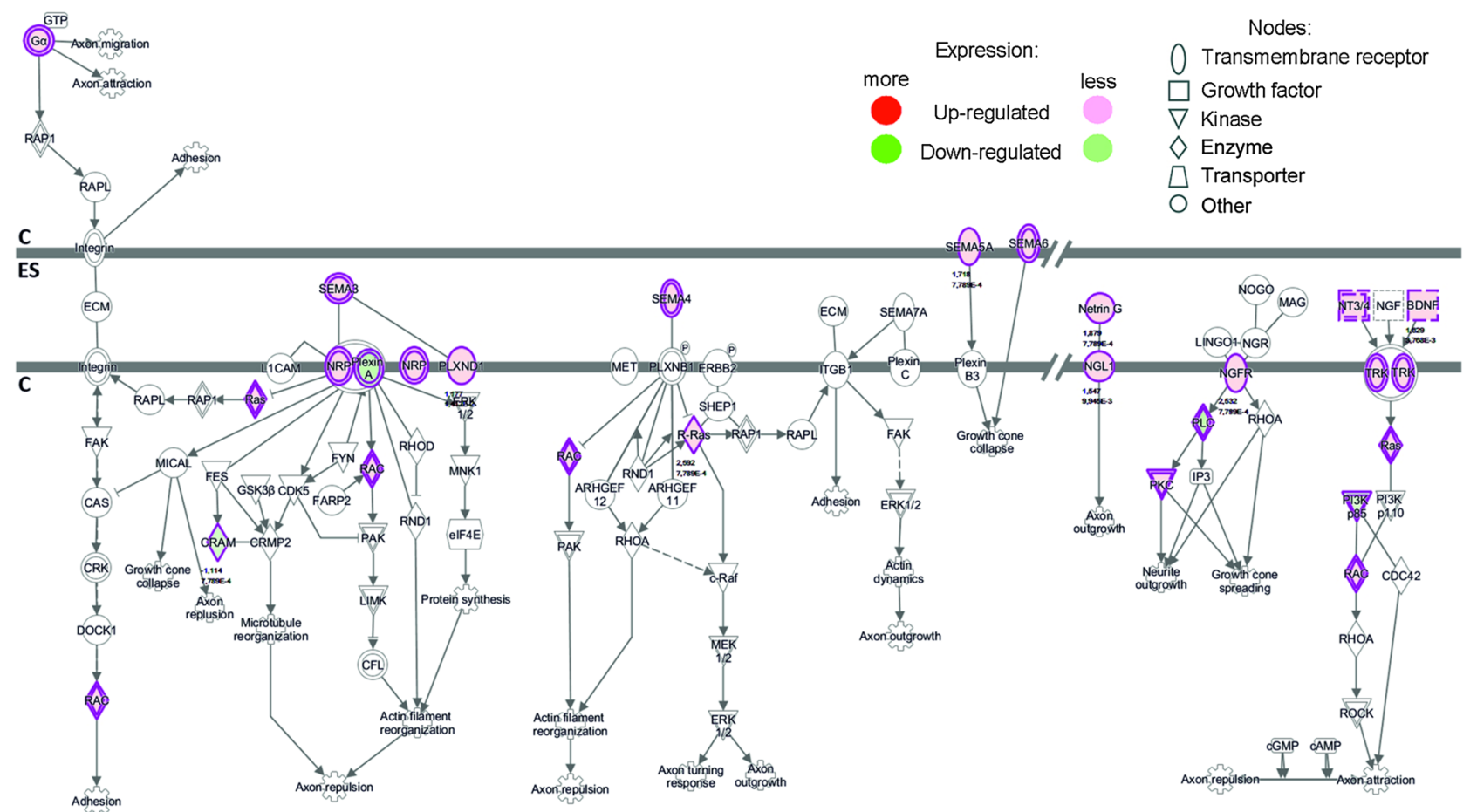

Fig. 2 Canonical pathway analysis. a Exemplification of most significantly enriched canonical pathways identified by the analysis of DEGs using IPA algorithm. The $-\log _{10}$ (B-H $p$ values) are presented as bars (upper axis), whereas triangles represent the percentage of overlap (defined as the percentage of a number of genes which meet the cut-off criteria over the total number of genes which constitute the pathway; lower axis). The number of differentially expressed genes assigned to different canonical pathways is shown. Axonal guidance signalling was significantly over-represented, with 73 DEGs assigned and an overlap equal to $16.9 \%$ (see Online Resource ESM 7 for the complete list of DEGs belonging to this pathway). b A graphical representation of axonal guidance signalling pathway. DEGs constituting this pathway encompassed cell surface receptors, extracellular growth factors and intra-cytoplasmic adapter proteins. The downstream functions related to the integrated neurite and axonal outgrowth, attraction and repulsion as well actin and tubulin cytoskeletal rearrangements. Several members of semaphorin family were differentially expressed in RA-NBM cells and influenced cytoskeletal and axonal remodelling processes through membrane receptors of plexin family. Moreover, an increased expression of $N G F R$ and $\operatorname{TrkB}$ (NTRK2) and related ligands (NTF4 and BDNF) impacted on axonal attraction/repulsion dynamics. Cellular compartments: $C$ cytoplasm, $E S$ extracellular space. Node borders: single borders delineate a network node with one member; double borders correspond to a node with several members (i.e., within TRK node, NTRK1, NTRK2 and NTRK3 are retained, of which NTRK2 was upregulated). c Depiction of significantly enriched functional categories encompassing axonal guidance signalling ( $\log _{2} \mathrm{FC} \geq+2$ and $\log _{2} \mathrm{FC} \leq-2$; Canonical pathway). The categories of neuritogenesis, formation of cellular protrusions and reorganization of cytoskeleton were all statistically enriched within the network module. The DEGs, which were further analysed on the protein level are marked with asterisk. Physical interaction between NTF4 and NTRK2 (BioGRID ID 301190), NRP1 and SEMA3A (BioGRID ID 883184) and NRP1 and SEMA3F (BioGRID ID 317335) are visualized. The $\log _{2} \mathrm{FC}$ values are indicated. Another network linking neuritogenesis, formation of cellular protrusions and axonogenesis is reported in Fig. S2 in Online Resource ESM 1 
known as NTRK2, the neurotrophic tyrosine kinase receptor Type 2). Through their convergence in common downstream functions, these molecules influence the balance between attraction and repulsion of axons, as well as other essential mechanisms of axonal guidance (Fig. 2b). Most of these DEGs were also shared within functional annotations identified by downstream effects analysis (Table 1 and Online Resource ESM 8). Accordingly, a small network module associated with axonal guidance signalling was identified with upregulated nodes linked mainly to semaphorins (SEMA3A, SEMA3D and SEMA3F and related receptor family member, NRPI) and neurotrophin signalling (NTRK2, NTF4). Further query of DEGs with $\log _{2} \mathrm{FCl}>2$ revealed the association to processes encompassing the neuronal differentiation, including neuritogenesis, formation of cellular protrusions and reorganization of cytoskeleton (Fig. 2c and Fig. S2 in Online Resource ESM 1). Other identified molecules in this

Table 1 Selected list of differentially expressed genes shared among functional annotations highlighted in downstream effects analysis and axonal guidance signalling pathway

\begin{tabular}{|l|c|c|c|c|c|c|c|}
\hline & \multicolumn{2}{|c|}{ Cytoskeleton } & \multicolumn{2}{c|}{ Plasma membrane } & Neuronal processes & $\begin{array}{c}\text { Canonical } \\
\text { pathway }\end{array}$ \\
\hline Gene & $\begin{array}{c}\text { Organization } \\
\text { of } \\
\text { cytoskeleton }\end{array}$ & $\begin{array}{c}\text { Microtubule } \\
\text { dynamics }\end{array}$ & $\begin{array}{c}\text { Formation of } \\
\text { plasma } \\
\text { membrane } \\
\text { projections }\end{array}$ & $\begin{array}{c}\text { Growth of } \\
\text { plasma } \\
\text { membrane } \\
\text { projections }\end{array}$ & Neuritogenesis & $\begin{array}{c}\text { Growth } \\
\text { of } \\
\text { neurites }\end{array}$ & $\begin{array}{c}\text { Axonal } \\
\text { guidance } \\
\text { signalling }\end{array}$ \\
\hline BDNF & $\bullet$ & $\bullet$ & $\bullet$ & $\bullet$ & $\bullet$ & $\bullet$ & $\bullet$ \\
\hline BMP7 & $\bullet$ & $\bullet$ & $\bullet$ & $\bullet$ & $\bullet$ & $\bullet$ & $\bullet$ \\
\hline DCC & $\bullet$ & $\bullet$ & $\bullet$ & $\bullet$ & $\bullet$ & $\bullet$ & $\bullet$ \\
\hline NGFR* & $\bullet$ & $\bullet$ & $\bullet$ & $\bullet$ & $\bullet$ & $\bullet$ & $\bullet$ \\
\hline NRP1 & $\bullet$ & $\bullet$ & $\bullet$ & $\bullet$ & $\bullet$ & $\bullet$ & $\bullet$ \\
\hline NTF4 & $\bullet$ & $\bullet$ & $\bullet$ & $\bullet$ & $\bullet$ & $\bullet$ & $\bullet$ \\
\hline NTNG1 & $\bullet$ & $\bullet$ & $\bullet$ & & $\bullet$ & & $\bullet$ \\
\hline NTRK2* & $\bullet$ & $\bullet$ & $\bullet$ & $\bullet$ & $\bullet$ & $\bullet$ & $\bullet$ \\
\hline PLXNA4* & $\bullet$ & $\bullet$ & $\bullet$ & $\bullet$ & $\bullet$ & $\bullet$ & $\bullet$ \\
\hline SEMA3A* & $\bullet$ & $\bullet$ & $\bullet$ & $\bullet$ & $\bullet$ & $\bullet$ & $\bullet$ \\
\hline SEMA3D & $\bullet$ & $\bullet$ & $\bullet$ & $\bullet$ & $\bullet$ & $\bullet$ & $\bullet$ \\
\hline SEMA3F & $\bullet$ & $\bullet$ & $\bullet$ & $\bullet$ & $\bullet$ & $\bullet$ & $\bullet$ \\
\hline WNT5A & $\bullet$ & $\bullet$ & $\bullet$ & $\bullet$ & $\bullet$ & $\bullet$ & $\bullet$ \\
\hline
\end{tabular}

Functional annotations are sorted for cellular compartments; selected DEGs, whose gene products were further analysed using semi-quantitative immunoblotting analysis, are marked by asterisks. Gene-NCBI gene symbol 
pathway included WNT family members and their related "frizzled" receptors as well as three members of the Bone Morphogenetic Proteins family (Online Resource ESM 7), which were also shared with the human embryonic stem cell pluripotency pathway (Fig. S3 and Supplementary Table in Online Resources ESM 1 and ESM 9, respectively).

\section{Expression of DEGs Products}

In order to confirm observed changes at the mRNA level, we assessed the expression of proteins encoded by DEGs assigned to both neuritogenesis and axonal guidance signalling pathway by immunoblotting analysis (Online Resources ESM 7, 8 and 9). We selected four highly upregulated DEGs $\left(\log _{2} \mathrm{FC}>2\right.$, including $N G F R, N T R K 2$, SEMA $3 A$ and SLITRKO) and one down-regulated DEG belonging to Plexin family (PLNXA4, $\log _{2} \mathrm{FC}<-2$ ), known to be a co-receptor for SEMA3A (Fig. 3a). In cell lysates, the expression of NTRK2/TrkB, NGFR and SLITRK6 was markedly increased in the differentiated $\mathrm{SH}$ SY5Y (Fig. 3b-d). Moreover, WB analysis of SEMA3A revealed two immunoreactive bands running approximately at 80 and $65 \mathrm{kDa}$; the latter one corresponded to a secreted and active form of SEMA3A, and it was the only isoform increased in RA-NBM cells (Fig. 3e). We did not find difference in PLNXA4 protein expression, although a significant downregulation upon differentiation was observed on mRNA level by qRT-PCR, confirming the results of RNA-seq analysis (Fig. 3f).

\section{Expression of Neuronal Markers}

To further validate the biological functions highlighted by IPA analysis, we investigated morphological changes and expression of neuronal markers by bright-field and immunofluorescence microscopy and WB.

Following 6 days of differentiation with RA, SH-SY5Y cells demonstrated decreased cellular density as compared to cells grown under basal conditions. Moreover, a subgroup of cells changed their morphology and acquired an elongated shape. These features did not change if the RA treatment was prolonged for another 3 days (data not shown). Conversely, cells exposed to the combined RANBM treatment exhibited remarkable changes in morphology, while many cells acquired a triangular neuronal shape and developed elongated processes with multiple branches although several flattened cells were visible (Fig. 4a). Furthermore, the results of immunofluorescent staining with antibodies against intermediate filaments and neuronal markers were in accordance with morphological features, characteristic for the RA-NBM differentiated cells towards a neuronal-like phenotype. In particular, extended neurites and branches were immunolabelled by $\beta$-III tubulin, while several elongated projections expressed phosphorylated medium-chain neurofilament proteins (pNF-M) along and at the termination of these structures. MAP2 staining was mainly restricted to cell bodies and faint staining could be observed for some axons. Conversely, a meaningful reduction of cell size and signal intensity of these markers was detected following 6 days of RA treatment alone, with a prompt recovery following introduction of NBM medium for another 3 days. The immunofluorescent signal of nestin, an intermediate filament precursor expressed in neuronal cell precursor, was unchanged in all tested culture conditions (Fig. 4b-c).

Immunoblotting analysis revealed increased signals for all cell markers in the presence of RA-NBM, which was statistically significant for pNF-M ( $p<0.01$, Fig. 5a, c). Different behaviours were observed for each marker according to specific growth conditions. For example, for the antibody recognizing the high molecular weight isoforms (MAP2A and MAP2B, of $\sim 200-250 \mathrm{kDa}$ ) and strongly the low molecular weight isoform (MAP2C, of $\sim 70 \mathrm{kDa}$ ), the signal intensity increased over time; however, the presence of RA in the medium reduced/altered the signal for all MAP2 isoforms (Fig. 5a). Cellular expression of nestin was detected at any time of observation, and it was enhanced by the presence of RA in the medium and specifically in RA-NBM differentiated cells. No change in the expression of intermediate filament marker vimentin was detected in any cell culture conditions tested (Fig. 5a).

Besides analysis of cytoskeletal proteins, we ascertained the expression of synapsin I, a marker of synaptic vesicles. In untreated cells, a sparse cytoplasmic pattern of staining was observed, whereas in RA-NBM differentiated cells the expression of synapsin I was localized along the axons in a spatial relationship with axonal varicosities, and at the flattened terminal portions (Fig. 6a-d). Moderate, but meaningful increase of the $75-\mathrm{kDa}$ band of synapsin I was detected following immunoblotting ( $p<0.05$, Fig. 6e-f).

\section{Elongation of Axonal Processes}

We used quantitative morphometric analysis to evaluate the length and numbers of pNF-H immunolabelled processes in RA-NBM differentiated cells (Fig. 7a). No meaningful difference in the number of primary axonal processes between cell grown in basal medium and differentiated cells was observed, whereas the number of secondary processes was significantly increased in RA-NBM differentiated cells (3.3/100 nuclei versus $0.4 / 100$ nuclei for undifferentiated cells, $p<0.05$; Fig. 7b). Moreover, few tertiary processes could be identified in differentiated cells only. Although the number of primary structures was not significantly changed, RA-NBM differentiated cells exhibited longer primary 
Fig. 3 Semi-quantitative Western blot analysis of selected DEGs products. a Five selected transcripts found differentially expressed in RANBM cells and their relative expression values (FPKM) are reported. The corresponding DEGs, assigned either to neuritogenesis or to axonal guidance signalling (or both, using canonical pathway IPA annotation) exhibited a $1 \log _{2} \mathrm{FCl}$ $\geq 2$, which corresponds to an absolute fold change in expression $\geq 4$.

b-e Immunoblotting analysis confirmed an up-regulation of TrkB, NGFR and SLITRK6. An increased expression of secreted $65 \mathrm{kDa}$ SEMA3A isoform was also detected. f Plexin A4 protein (WB) and mRNA (qRTPCR) expression analyses. No significant change in protein expression was observed (left panel); a significant downregulation of PLXNA4 mRNA levels was found in RANBM differentiated cells, in accordance with the results of RNA-seq analysis (absolute fold change $=-2.4$ which corresponds to

$\log 2 \mathrm{FC}=-1.28$; right panel). GAPDH served as internal standard, a.u. arbitrary units; $* p<0.05, * * * p<0.001$ mean \pm s.e.m. of four independent experiments

A

\begin{tabular}{|clcccc|}
\hline Gene & \multicolumn{1}{c}{ Gene Name } & $\begin{array}{c}\text { FPKM } \\
\text { NT }\end{array}$ & $\begin{array}{c}\text { FPKM } \\
\text { RA-NBM }\end{array}$ & Log $_{2}$ FC & q-value \\
NTRK2 & BDNF/NT-3 Growth Factors Receptor & 0.69 & 39.76 & 5.85 & $7.79 \mathrm{E}-04$ \\
NGFR & Nerve Growth Factor Receptor & 1.25 & 7.24 & 2.53 & $7.79 \mathrm{E}-04$ \\
SEMA3A & Semaphorin-3A & 11.19 & 163.17 & 3.87 & $7.79 \mathrm{E}-04$ \\
SLITRK6 & SLIT And NTRK-Like Family, Member 6 & 0.06 & 3.66 & 5.92 & $7.79 \mathrm{E}-04$ \\
PLXNA4 & Plexin-A4 & 17.52 & 3.93 & -2.16 & $7.79 \mathrm{E}-04$ \\
\hline
\end{tabular}

B
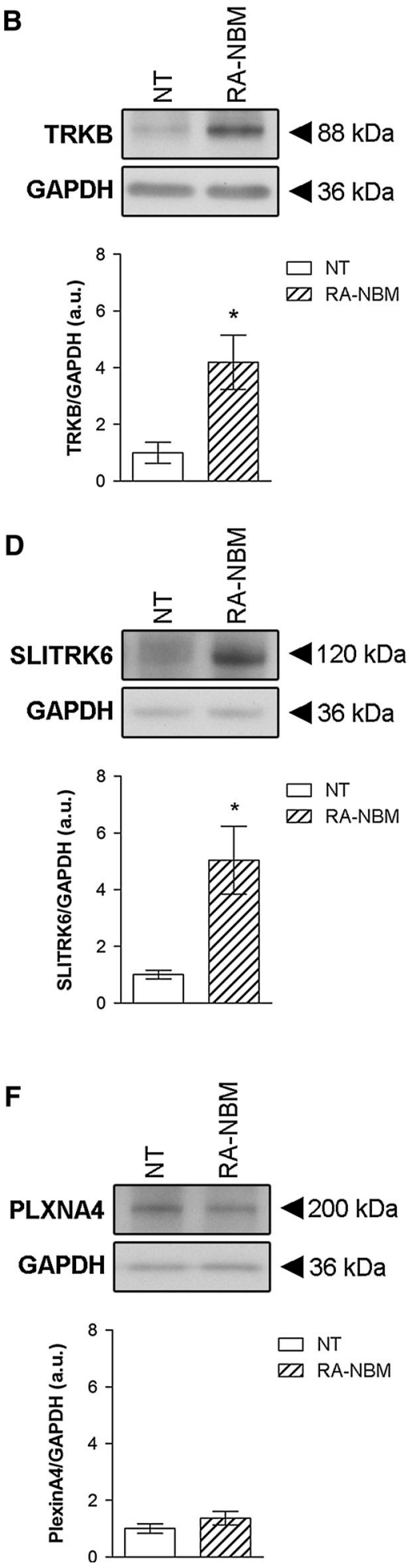
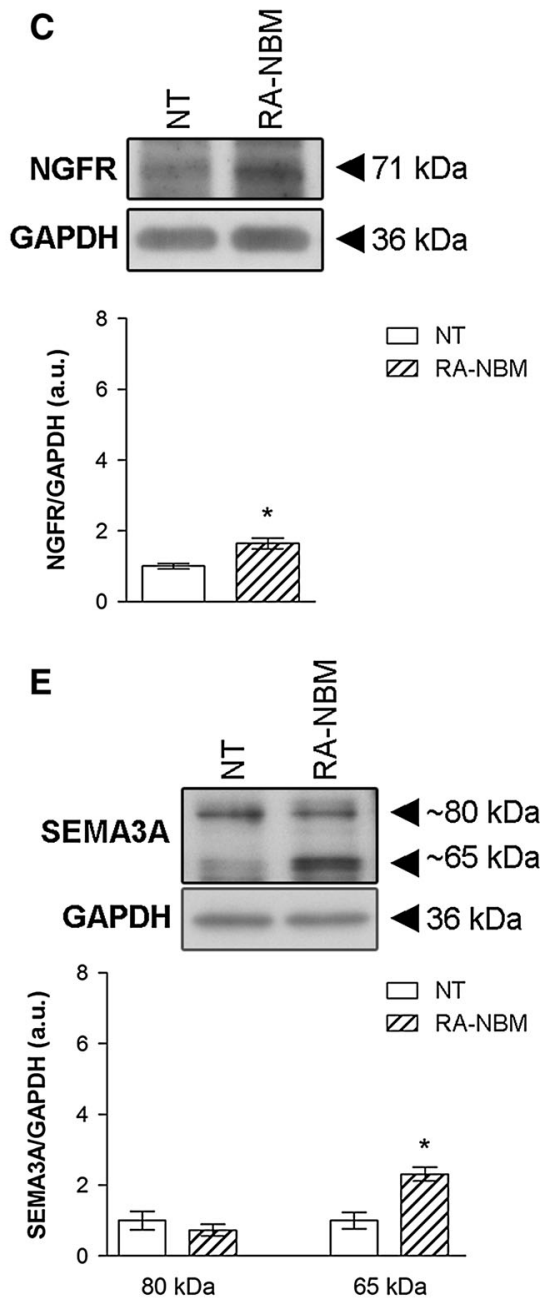

qRT-PCR

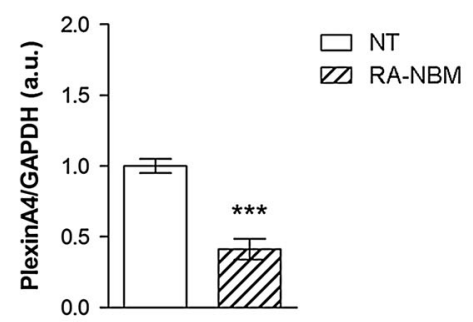


A

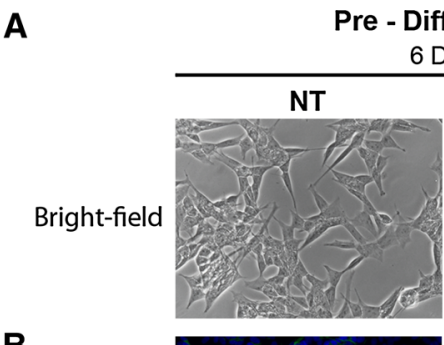

B

$\beta$-III tubulin
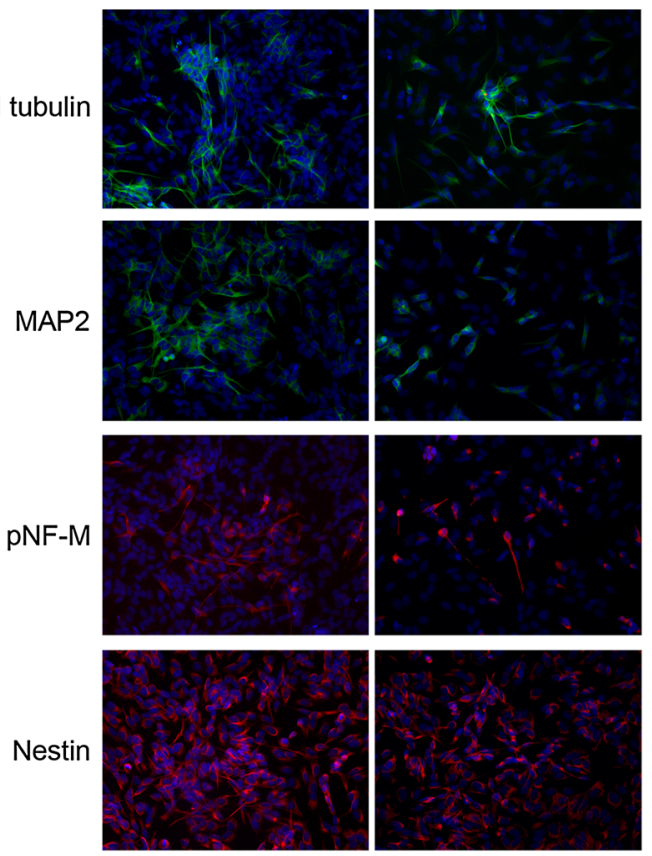

C

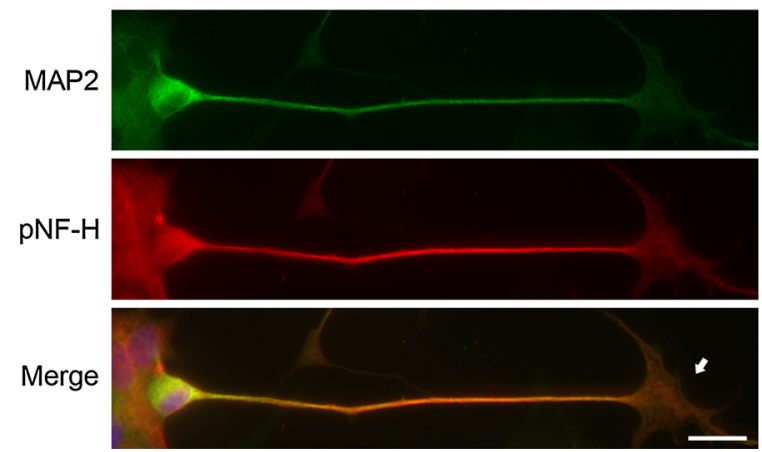

Fig. 4 Morphological analysis of differentiated SH-SY5Y cells. a At 6-DIV stage, the cells exposed to RA showed an elongated morphology as compared to basal medium (NT). Cells subsequently treated in NBM for 3 days became more polarized, exhibited several neurites and branches and acquired a neuronal-like shape. Scale bar $20 \mu \mathrm{m}$. b The morphological changes were associated with a remarkable expression of neuronal cytoskeletal markers, such as $\beta$ III tubulin, MAP2 and phosphorylated medium-chain neurofilament (pNF-M, BF10 antibody). Intense MAP2 immunofluorescent staining was observed in NT cells at 9 DIV, and can be associated with high

processes as compared to controls (mean length 87.35 versus $48.62 \mu \mathrm{m}, p<0.01$; Fig. $7 \mathrm{c}$ ). Interestingly, a shift in the length of such structures between the two cell populations was observed. In particular, a higher percentage of
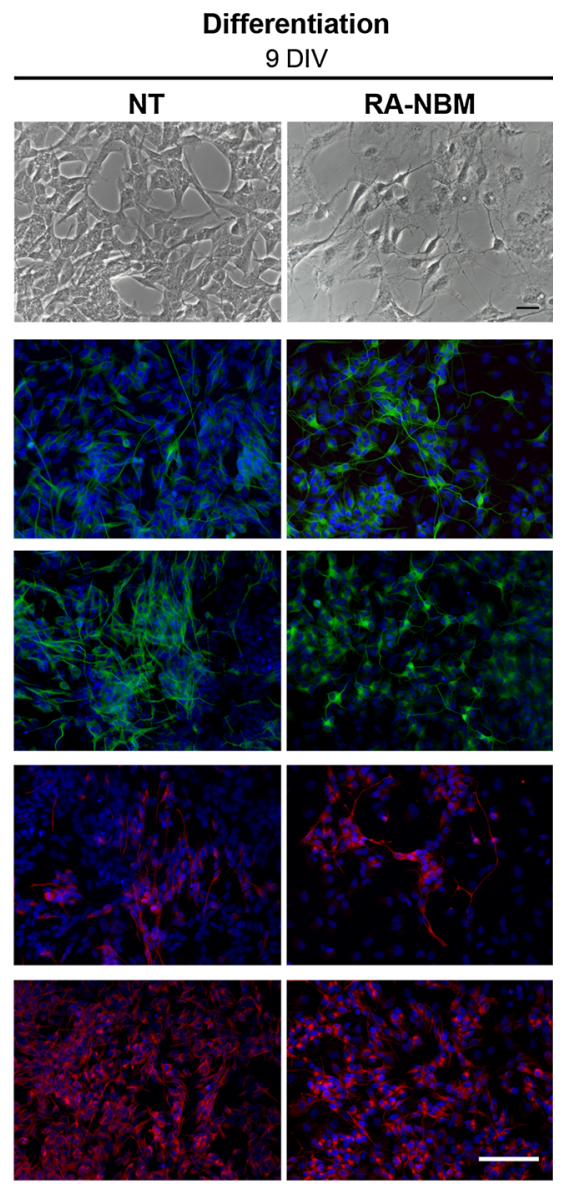

cell density. No significant difference in nestin expression between basal and NBM differentiated cultures was found. DIV days in vitro, scale bar $100 \mu \mathrm{m}$. c Process of an axonal sprouting from a cell body detected in RA-NBM differentiated culture is visualized. MAP2 staining is localized to the cell body and proximal portion of the axon, and a fainter signal is also detected and along the axon; intense axonal labelling of phosphorylated heavy-chain neurofilaments (pNF-H) is also observed at the termination zone (growth cone, arrow). Scale bar $20 \mu \mathrm{m}$

shorter processes (ranging 30-90 $\mu \mathrm{m}$ ) was found in the untreated cells $(p<0.05)$, whereas the longest processes $(>90 \mu \mathrm{m})$ were most visible in RA-NBM treated cells $(p<0.05$; Fig. 7d). 


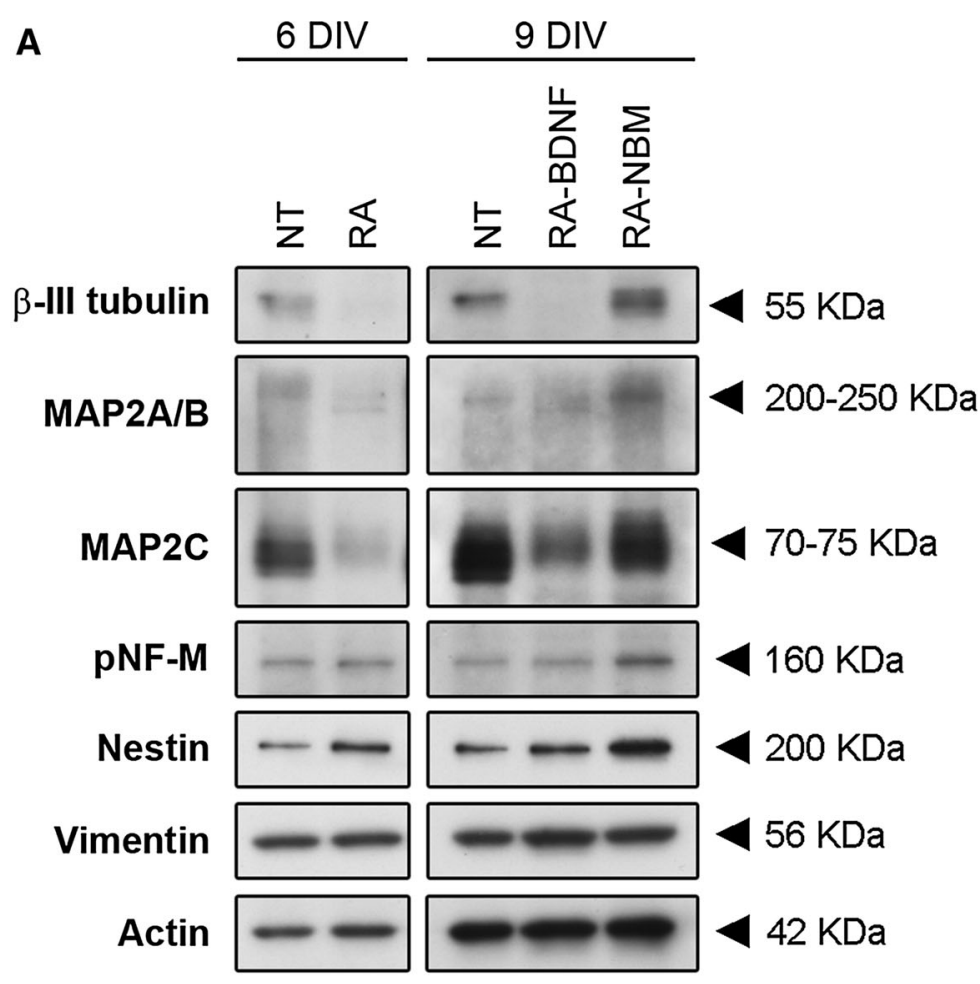

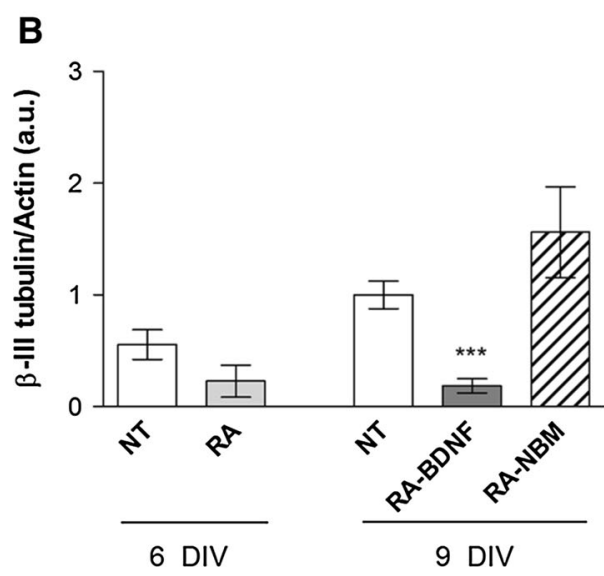

Fig. 5 Qualitative and semi-quantitative Western blotting analysis of neuronal cytoskeletal proteins in SH-SY5Y cells under different growth conditions. a SH-SY5Y cells differentiated in RA-NBM showed an enhanced expression of $\beta$-III tubulin and phosphorylated medium-chain neurofilaments (pNF-M, BF-10 antibody) as compared to neuroblastoma cells cultured in basal, RA and RA-BDNF media. High molecular weight isoforms of MAP2 (A/B) were expressed in RA-NBM treated cells, whereas no significant change for low molecular isoforms (MAP2C) was detected. Notably, an increased expression of nestin, a neuronal marker of early-differentiation stage

\section{Discussion}

It has been known for a long time that SH-SY5Y cells grown in RA-containing medium and/or other enriched media can switch from an undifferentiated state towards a more mature phenotype (Arcangeli et al. 1999; Encinas

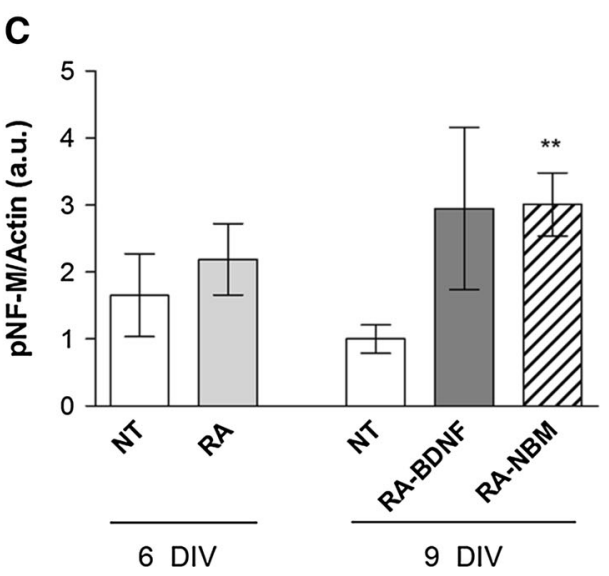

was observed following both RA and RA-NBM treatment whereas the expression of vimentin, a marker specific of mesenchymal lineage remained unaltered in all of the experimental conditions. b, c Semiquantitative evaluation of $\beta$-III tubulin and pNF-M confirmed an increased expression of both markers in RA-NBM cell lysates as compared to NT (9 DIV) samples, even though the statistical significance was evident for pNF-M only. Conversely, a decreased expression of $\beta$-III tubulin was observed in RA and RA-BDNF lysates. $D I V$ days in vitro, $* * p<0.01, * * * p<0.001 ; a . u$. arbitrary units; mean \pm s.e.m. of three independent experiments

et al. 2000; Pahlman et al. 1984). In fact these cells decrease their proliferative rate, reduce their tendency to undergo cell death, change their outer profile acquiring a neuronal-like shape, and eventually promote outgrowth of cytoplasmic processes to become an elongated axon (Constantinescu et al. 2007; Dwane et al. 2013; Encinas 

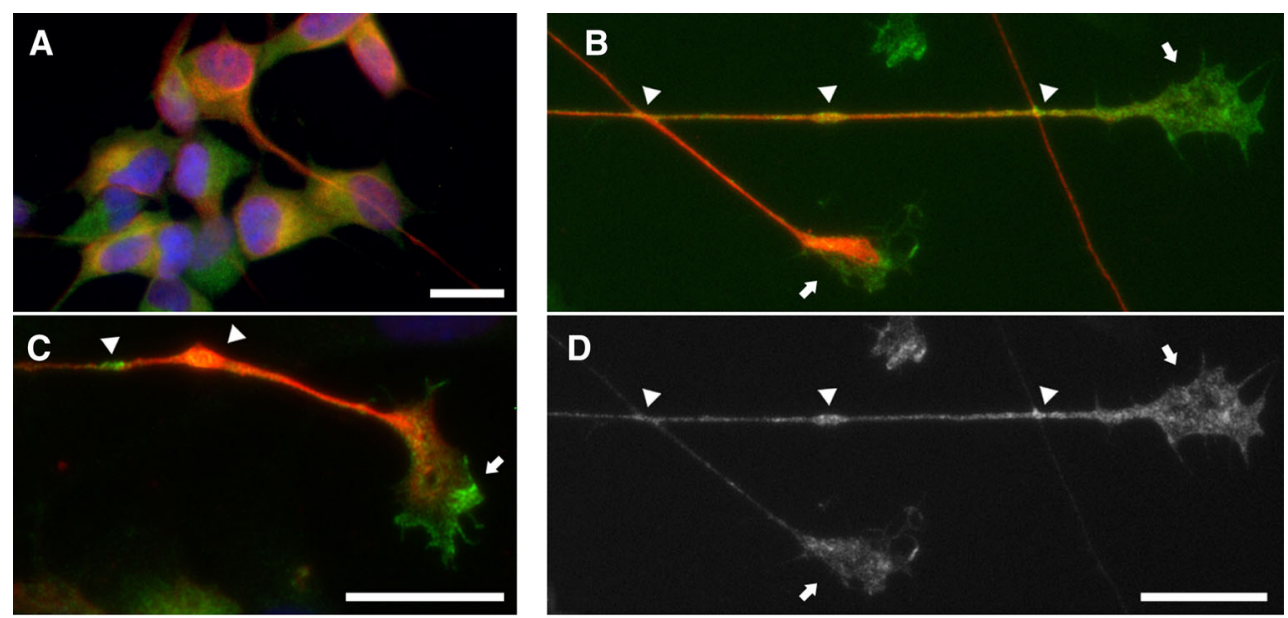

$\mathbf{E}$

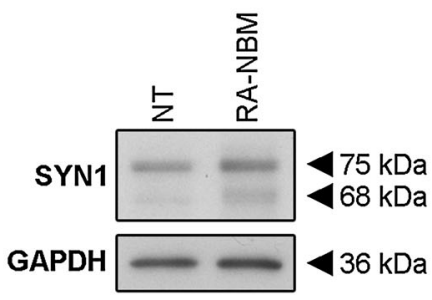

$\mathbf{F}$

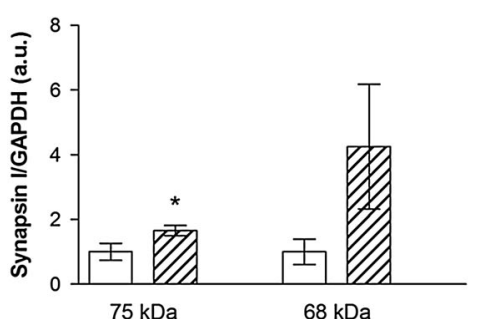

$\square$ NT

$\square Z$ RA-NBM
Fig. 6 Immunofluorescence and immunoblotting analyses of synapsin I. a-d Double immunostaining for synapsin I (green) and pNF-H (red) of both NT and RA-NBM differentiated SH-SY5Y cells. Morphologically, parental cells were characterized by a sparse cytoplasmic pattern of staining for synapsin I (a), whereas in RANBM treated cells the immunostaining was mainly localized along the elongated processes and at the termination zone (growth cones) of

et al. 2000; Kovalevich and Langford 2013). It is plausible that sequential and coordinated activities of different classes of molecules under the control of several sets of genes, differentially regulated by the growth conditions, are necessary to sustain the morphological features and behaviour of differentiated SH-SY5Y cells.

The analysis of RNA-seq data provided several clues to understand molecular mechanisms associated with the differentiation process. Categorization with IPA suggested that functional annotations related to the general remodelling of cellular shape (i.e., formation and outgrowth of plasma membrane projections, organization of the cytoskeleton and microtubule dynamics) are key events in differentiation. Formation of cellular protrusions (lamellipodia and filopodia) in neuronal cells is crucial for the development and guidance of nerve growth cones (Vitriol and Zheng 2012). Accordingly, the functional annotations that we scrutinized in more detail related with the early steps of neurite extension, and we observed that 73 DEGs constituted the axonal guidance signalling pathway. This pathway is composed of molecules which are localized outside the cell (extracellular soluble factors), on the plasma membrane surface (receptors) or inside the the same structures (pointed by arrowheads and arrows, respectively; b-d). Scale bars $20 \mu \mathrm{m}$. e, f Following immunoblotting analysis, a moderate change in the expression of $\sim 75 \mathrm{kDa}$ band as well as a more intense (although not statistically significant) signal at a $\sim 68 \mathrm{kDa}$ were detected in RA-NBM differentiated cells. a.u. arbitrary units; ${ }^{*} p<0.05$; mean \pm s.e.m. of three independent experiments (Color figure online)

cytoplasm (adapter signalling molecules and cytoskeletal proteins; Fig. 2, Thiede-Stan and Schwab 2015). All these molecules coordinate the cellular mechanisms, which drive growth cones and cellular protrusions through the extracellular matrix and towards the cellular targets. In particular, during the pathfinding process this cohort of soluble factors and receptors jointly regulates elongation of axonal structures, adhesion to the extracellular matrix, cytoskeletal remodelling as well as the attraction/repulsion balance among growing axons (Bearce et al. 2015; Myers et al. 2011). Among the DEGs assigned to this pathway and to the aforementioned functional annotations (Table 1 and Online Resource ESM 8) we identified several classes of genes which are known to play a pivotal role in specific neuronal functions, such as growth, differentiation and neurite outgrowth. We observed an up-regulation in transcript expression of two neurotrophins (BDNF and NTF4) and of two related receptors (NTRK2 and NGFR), as well as a direct relationship between gene and associated protein expression. Following immunoblotting analysis, we detected an increased amount of the proteins encoded by NGFR and NTRK2 genes. These findings were consistent with previous reports, which demonstrated that exposure to 


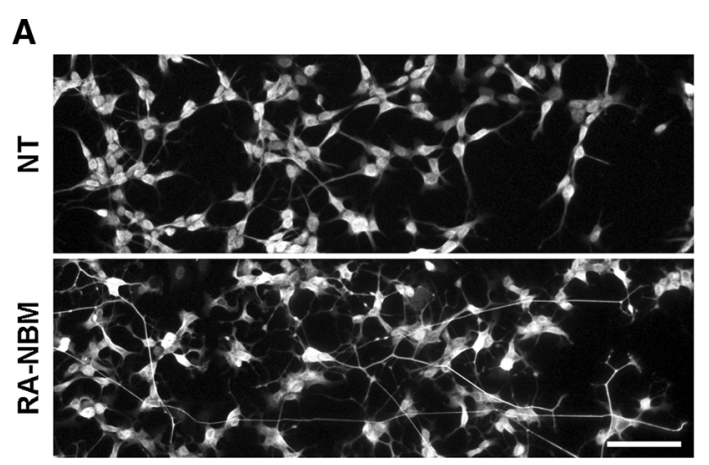

B

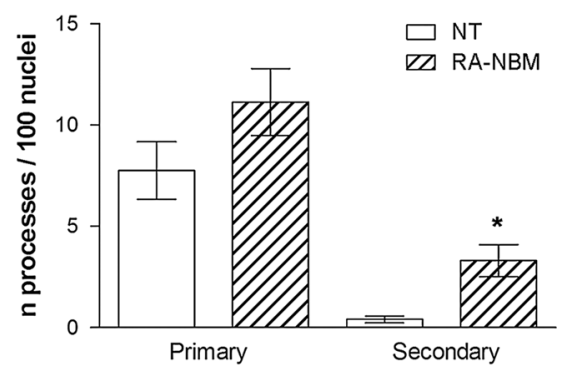

C

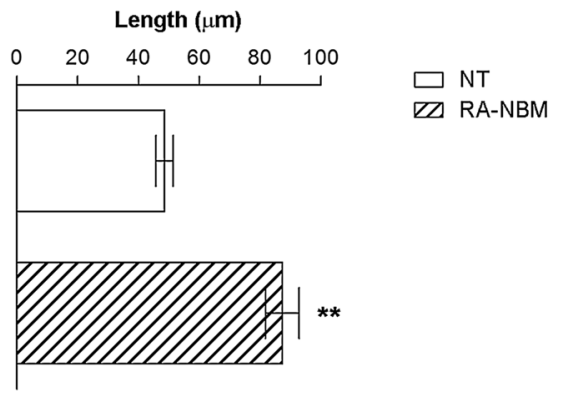

Fig. 7 Quantitative analysis of SMI31-immunolabelled processes in non-differentiated and differentiated cells. a Immunofluorescent staining of phosphorylated heavy chain neurofilaments (pNF-H; SMI-31R antibody) showed elongated processes in RA-NBM differentiated cells, in comparison to the cells grown in basal medium (nondifferentiated cells, NT). Scale bar $100 \mu \mathrm{m}$. b Quantitative analysis was performed on immunolabelled structures, traced using NeuronJ. Normalization was performed on 100 cells (see "Materials and Methods" for further details). The number of primary processes was slightly increased in differentiated cells (without statistical

RA both increases the expression of NTRK2/TrkB in neuroblastoma cells and improves the responsiveness to the exogenous ligand (Kaplan et al. 1993; Middlemas et al. 1999; Shiohira et al. 2012). Furthermore, we found a remarkably increased expression of SLITRK6 transcript and of the associated encoded protein. SLITRK6 belongs to the SLITRK gene family, which encodes transmembrane proteins prevalently expressed in neuronal tissues. These proteins show structural homology with Trk receptors and are involved in neurite outgrowth (Aruga and Mikoshiba 2003; Aruga et al. 2003). Expression of SLITRK genes and related proteins has not been previously reported in neuroblastoma cells following differentiation. Interestingly, another member of this family, SLITRK3, was found differentially expressed in RA-NBM cells and linked to neuritogenesis annotation (Online Resource ESM10). Recently, SLITRK5 was shown to modulate BDNF-dependent biological responses by regulating TrkB receptor recycling (Song et al. 2015). Whether the increased expression of SLITRK6/SLITRK6 observed in this experimental setting (Fig. 3d) can be associated with the enhanced expression of BDNF and NTRK2/TrkB, and to a
D

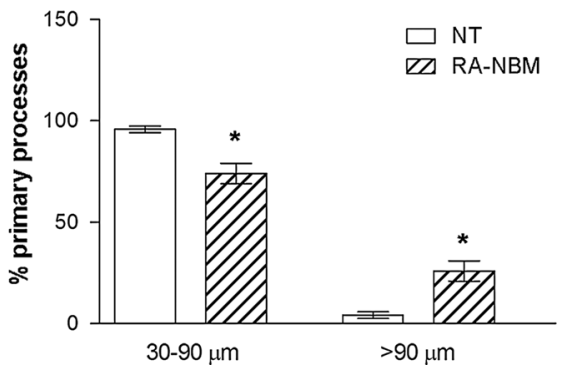

significance). On the contrary, a number of secondary processes which branched from a primary structure were increased on RA-NBM treated cells. c Primary processes were significantly longer in differentiated cells versus untreated cells. $\mathbf{d}$ Increased percentage of primary processes longer than $90 \mu \mathrm{m}$ was detected on RA-NBM treated cells as compared to basal conditions, whereas shorter primary processes were more evident in cells grown in basal culture medium. ${ }^{*} p<0.05$, ${ }^{* *} p<0.01$; mean \pm s.e.m. of four independent experiments

putative autocrine loop stimulated by rhBDNF in RA-NBM requires further investigations.

Other noteworthy differentially expressed genes assigned to the axonal guidance signalling pathway were members of the semaphorin family and several genes encoding for BMP and WNT proteins (Online Resource ESM 7). Specifically, we identified transcriptional changes for many semaphorin (SEMA3A, 3D, 3F, 4G, 5A, 6A, 6C, and $6 D)$ and related plasma membrane receptors (PLXNA2, $P L X N D 1$ and NRP1). Semaphorins exert their effects by finely regulating the balance between attraction and repulsion of growing axons in neuronal cells, as reported in both in vitro and in vivo models (Thiede-Stan and Schwab 2015; Zhou et al. 2008). We ascertained the expression of SEMA3A protein by immunoblotting and detected an increased expression of the secreted $65-\mathrm{kDa}$ isoform (Antipenko et al. 2003; Tran et al. 2007). SEMA3A was reported to inhibit axonal growth and stimulate dendritic formation as well as the axonal transport (Mann and Rougon 2007), thus influencing the polarization of neuronal cells and the pathfinding process (Shelly et al. 2011). SEMA3A exerts its effects upon binding with heteromeric 
complexes formed by a member of neuropilin receptor family (neuropilin-1/NRP1) and a member of type-A Plexin family (Plexin A4/PLXNA4) (Mlechkovich et al. 2014; Yaron et al. 2005). Interestingly, different behaviour was observed for both SEM3A receptors, namely PLNXA4 transcript was down-regulated while NRP1 was up-regulated, although the expression of PLXNA4 protein was not significantly changed. The downregulation of PLXNA4 mRNA levels was confirmed in quantitative PCR experiments, further supporting the established notion that transcript and protein expression level are not always strictly related (Koussounadis et al. 2015; Vogel and Marcotte 2012). The relative high number of DEGs encoding for semaphorins $(n=8)$ and their related receptors $(n=4)$ may account for the necessity of finely tuned mechanisms in order to accomplish the functions assigned to this class of molecules.

A key event in neuronal maturation is the ability to generate and elongate a primary axon and its branches. We have generated various lines of evidence that RA-NBM treated neuroblastoma cells turned into differentiated neurons. Immunolabelling of axons with microtubules ( $\beta$-III tubulin) and neurofilamentous proteins (pNF-M and pNF-H), displayed a classical pattern observed in mature axonal processes. Furthermore, the morphometric analysis revealed that axonal elongations occur more frequently in RA-NBM treated cells as compared to controls. In fact, a larger number of cells exhibited longer axons with an increased number of secondary structures. Immunolabelling of a synaptic marker, synapsin I demonstrated both scattered signals alongside the axons and a putative vesicular pattern at the terminal ends. These findings indicate that the neuronal machinery of the differentiated cells has been "instructed" to deliver synaptic material to the end-terminal, and possibly to create synaptic contacts. Biochemical investigations on expression of cytoskeletal markers in RA-NBM treated cells further provided hints on how to delineate the process of SH-SY5Y differentiation. Together with the presence of cells expressing markers of mature neurons ( $\beta$-III tubulin, pNF$\mathrm{M}$, and high molecular weight MAP2 isoforms, MAP2A and B) we found an increased expression of nestin, an early intermediate filaments marker detected in cells of multilineage progenitor cells (including neuronal progenitors; Wiese et al. 2004) as well as persistent, high expression of MAP2C, a low molecular weight isoform expressed during development in the axonal compartment only (Meichsner et al. 1993). In our view, these findings suggest that cell populations at different stages of differentiation (from stemlike, nestin-positive cells, to mature neuron-like elements with elongated, mature axons) are more represented in RANBM treated cultures than in controls.

Along with the cellular phenomena related to morphological and functional changes occurring during cell differentiation, the IPA algorithm further highlighted DEGs associated with cell proliferation, death and survival. The annotation proliferation of tumour cells lines was found to be significantly inhibited in RA-NBM treated cells, predicting a dramatic reduction of the high rate of cell proliferation classically observed in SH-SY5Y cells. Specifically, four down-regulated DEGs (namely, ID1, ID2, ID3 and ID4) encoded for transcription regulators, which were reported to be repressed during the neuronal differentiation of neuroblastoma cells in the presence of RA (Cheung et al. 2009; Lopez-Carballo et al. 2002). Furthermore, analysis of annotations assigned to cell death and survival gave either negative or positive predictions of cell death, cell survival or cell viability according to different cell types (Online Resource ESM 6D). Our preliminary observations using FACS analysis suggest an increased percentage of viable cells as well as a reduced percentage of dead cells (both early and late apoptotic cells evaluated by AnnexinV/PI assay) in RA-NBM cultures as compared to basal conditions (Fig. S4 in Online Resource ESM 1). Further investigations addressing the issues of cell proliferation and death are necessary to confirm these preliminary findings.

The canonical pathway analysis (Fig. 2b) revealed also other categories to be significantly overrepresented, including hepatic fibrosis and hepatic stellate cell activation $(-\log \mathrm{B}-\mathrm{H} p$ value $=9.32)$, human embryonic stem cell pluripotency $(-\log \mathrm{B}-\mathrm{H} p$ value $=4.4)$ and colorectal cancer metastasis signalling $(-\log \mathrm{B}-\mathrm{H} p$ value $=4.05)$. Interestingly, several DEGs were shared among categories, including for example 18 DEGs common for axonal guidance signalling and colorectal cancer metastasis signalling, and 16 DEGs shared with human embryonic stem cell pluripotency (Fig. S3 and Supplementary Table in Online Resources ESM 1 and ESM 9, respectively). Among DEGs shared between axonal guidance signalling, colorectal cancer metastasis signalling and human embryonic stem cell pluripotency, 4 members of WNT family were found (WNT5B, WNT9A, WNT10A, WNT11), while 3 members of PDGF and 2 of BMP family were apportioned to axonal guidance signalling and human embryonic stem cell pluripotency. These findings indicate that various cellular cascades crosstalk in SH-SY5Y differentiation, and are possibly related to distinct changes in the proliferation state and differentiation processes of the tumour cell line.

Several signatures identified in our experimental settings were related to various cellular events, including proliferation, self-renewal and commitment to a more mature phenotype, known hallmarks of the dynamics of cellular processes occurring during the differentiation of neuronal progenitor cells (NPCs). Notch signalling related proteins, WNTs, BMPs, frizzled receptors as well as GLI1 and inhibitor of DNA binding/differentiation (ID) proteins are 
only few examples from numerous group of molecular factors involved in the control of NPCs' fate (Christie et al. 2013). Members of these gene families were found to be differentially expressed in our RNA-seq analysis (Fig. S5 and Supplementary Table in Online Resources ESM 1 and ESM 7, respectively). Therefore, our experimental data indicate that SH-SY5Y cells are able to activate, at least in part, the same molecular machinery involved in the physiological neurogenesis, in order to prompt the decision to proliferate or differentiate. On the other hand, the results of top canonical pathways bioinformatic analyses pinpointed to other cellular processes, closely related to the features displayed by tumour cells.

Taken together, our transcriptomic and cellular analyses of SH-SY5Y cells revealed the simultaneous presence of proliferative, differentiating and death-related cellular events in RA-NBM cultures. Both morphological and biochemical data suggest that different subsets of cells are likely present in RA-NBM cultures: (1) differentiated neuronal-like cells, with long primary axons and multiple secondary and tertiary branches, expressing neuronal markers; (2) partially differentiated MAP2C-positive cells and "stem-like", nestin-labelled cells, which may still proliferate; (3) undifferentiated, tumour-like cells with predicted decreased proliferation rate. Such asynchronous differentiation has been recently reported in an experimental SH-SY5Y model, focusing on the role of Amyloid $\beta$ Precursor Protein, A $\beta$ PP in differentiation process (da Rocha et al. 2015).

The transcriptomic analysis performed in this study identified about 2000 DEGs associated with the differentiation of SH-SY5Y cells grown in a specifically enriched medium. Most DEGs were linked to functional annotations/modules and cellular pathways related to neuronal differentiation, and some gene families were recognized to be involved in this process for the first time (i.e., SLITRK and semaphorins). Furthermore, relatively low numbers of DEG were linked to other specific neuronal functions, such as neurotransmission and synaptic transmission (199 out of 1989 DEGs of the whole dataset; see Supplementary Table 5 in ESM 5). Related to this annotation, no clear trends of expression of checked DEGs and related annotations were noticed. The precise biological significance of most data remains unknown and requires more careful investigations. This is of particular relevance considering that differentiated SH-SY5Y cells are used as in vitro model to study cellular mechanisms involved in several human neurodegenerative disorders. Exposure to differentiation media may unsettle different cellular behaviours (such as the response to oxidative stress) and, therefore, the cell properties to cope with the selected stimuli, even affecting their survival capacities and/or vulnerability to cell death (Forster et al. 2016; Palomo et al. 2011; Schneider et al. 2011).

Acknowledgments Dr. A. Ferrarini and Dr. E. Dal Molin from the Department of Biotechnology of Verona University are fully acknowledged for their experienced support with RNA-seq analysis. We are grateful to the scientists and technicians of the Laboratory of Neuropathology, University of Verona School of Medicine. We also thank Dr. Enzo Scifo (University of Toronto, Canada) for critical comments on the manuscript.

Author contributions F.P: designed and performed experiments, interpreted data, prepared figures and tables, and wrote the manuscript; L.B.: performed experiments and analysed data; F.DiL.: analysed and interpreted data; M.B.: analysed data; E.Z.: performed experiments and analysed data; R.C.: analysed and interpreted data; M.D.: designed experiments, interpreted data, revised the manuscript; F.M.S.: conceived the study, and revised the manuscript; M.L. designed the study, analysed and interpreted data, prepared figures, revised the manuscript; A.S.: conceived and designed the study, analysed and interpreted data, drafted and revised the manuscript.

Funding This study was funded by European Union Seventh Framework Programme (FP7/2007-2013) under Grant Agreement No 281234.

Compliance with ethical standards

Conflict of interest The authors declare that they have no conflict of interest.

\section{References}

Agholme L, Lindstrom T, Kagedal K, Marcusson J, Hallbeck M (2010) An in vitro model for neuroscience: differentiation of SHSY5Y cells into cells with morphological and biochemical characteristics of mature neurons. J Alzheimers Dis 20:10691082. doi:10.3233/JAD-2010-091363

Agholme L, Nath S, Domert J, Marcusson J, Kagedal K, Hallbeck M (2014) Proteasome inhibition induces stress kinase dependent transport deficits-implications for Alzheimer's disease. Mol Cell Neurosci 58:29-39. doi:10.1016/j.mcn.2013.11.001

Antipenko A et al (2003) Structure of the semaphorin-3A receptor binding module. Neuron 39:589-598

Arcangeli A et al (1999) Modulation of HERG current and herg gene expression during retinoic acid treatment of human neuroblastoma cells: potentiating effects of BDNF. J Neurobiol 40:214-225

Aruga J, Mikoshiba K (2003) Identification and characterization of Slitrk, a novel neuronal transmembrane protein family controlling neurite outgrowth. Mol Cell Neurosci 24:117-129

Aruga J, Yokota N, Mikoshiba K (2003) Human SLITRK family genes: genomic organization and expression profiling in normal brain and brain tumor tissue. Gene 315:87-94

Batagov AO, Yarmishyn AA, Jenjaroenpun P, Tan JZ, Nishida Y, Kurochkin IV (2013) Role of genomic architecture in the expression dynamics of long noncoding RNAs during differentiation of human neuroblastoma cells. BMC Syst Biol 7(Suppl 3):S11. doi:10.1186/1752-0509-7-S3-S11

Bearce EA, Erdogan B, Lowery LA (2015) TIPsy tour guides: how microtubule plus-end tracking proteins (+TIPs) facilitate axon 
guidance. Front Cell Neurosci 9:241. doi:10.3389/fncel.2015. 00241

Bell N, Hann V, Redfern CP, Cheek TR (2013) Store-operated $\mathrm{Ca}(2+)$ entry in proliferating and retinoic acid-differentiated $\mathrm{N}$ and S-type neuroblastoma cells. Biochim Biophys Acta 1833:643-651. doi:10.1016/j.bbamcr.2012.11.025

Biedler JL, Roffler-Tarlov S, Schachner M, Freedman LS (1978) Multiple neurotransmitter synthesis by human neuroblastoma cell lines and clones. Cancer Res 38:3751-3757

Borriello A et al (2006) Retinoic acid induces p27Kip1 nuclear accumulation by modulating its phosphorylation. Cancer Res 66:4240-4248. doi:10.1158/0008-5472.CAN-05-2759

Cheung YT, Lau WK, Yu MS, Lai CS, Yeung SC, So KF, Chang RC (2009) Effects of all-trans-retinoic acid on human SH-SY5Y neuroblastoma as in vitro model in neurotoxicity research. Neurotoxicology 30:127-135. doi:10.1016/j.neuro.2008.11.001

Christie KJ, Emery B, Denham M, Bujalka H, Cate HS, Turnley AM (2013) Transcriptional regulation and specification of neural stem cells. Adv Exp Med Biol 786:129-155. doi:10.1007/97894-007-6621-1_8

Constantinescu R, Constantinescu AT, Reichmann H, Janetzky B (2007) Neuronal differentiation and long-term culture of the human neuroblastoma line SH-SY5Y. J Neural Transm Suppl:17-28

Cuende J, Moreno S, Bolanos JP, Almeida A (2008) Retinoic acid downregulates Rae1 leading to APC(Cdh1) activation and neuroblastoma SH-SY5Y differentiation. Oncogene 27:3339-3344. doi:10.1038/sj.onc. 1210987

da Rocha JF, da Cruz e Silva OA, Vieira SI (2015) Analysis of the amyloid precursor protein role in neuritogenesis reveals a biphasic SH-SY5Y neuronal cell differentiation model. J Neurochem 134:288-301. doi:10.1111/jnc.13133

de Bittencourt Pasquali MA et al (2016) Gene expression profile of NF-kappaB, Nrf2, glycolytic, and p53 pathways during the SHSY5Y neuronal differentiation mediated by retinoic acid. Mol Neurobiol 53:423-435. doi:10.1007/s12035-014-8998-9

Dwane S, Durack E, Kiely PA (2013) Optimising parameters for the differentiation of SH-SY5Y cells to study cell adhesion and cell migration. BMC Res Notes 6:366. doi:10.1186/1756-0500-6-366

Encinas M et al (2000) Sequential treatment of SH-SY5Y cells with retinoic acid and brain-derived neurotrophic factor gives rise to fully differentiated, neurotrophic factor-dependent, human neuron-like cells. J Neurochem 75:991-1003

Ferreira PS et al (2013) Neurotoxicity of "ecstasy" and its metabolites in human dopaminergic differentiated SH-SY5Y cells. Toxicol Lett 216:159-170. doi:10.1016/j.toxlet.2012.11. 015

Forster JI et al (2016) Characterization of differentiated SH-SY5Y as neuronal screening model reveals increased oxidative vulnerability. J Biomol Screen. doi:10.1177/1087057115625190

Gimenez-Cassina A, Lim F, Diaz-Nido J (2006) Differentiation of a human neuroblastoma into neuron-like cells increases their susceptibility to transduction by herpesviral vectors. J Neurosci Res 84:755-767. doi:10.1002/jnr.20976

Goldie BJ, Barnett MM, Cairns MJ (2014) BDNF and the maturation of posttranscriptional regulatory networks in human SH-SY5Y neuroblast differentiation. Front Cell Neurosci 8:325. doi:10. 3389/fncel.2014.00325

Hadzhieva M et al (2013) Dysregulation of iron protein expression in the G93A model of amyotrophic lateral sclerosis. Neuroscience 230:94-101. doi:10.1016/j.neuroscience.2012.11.021

Jamsa A, Hasslund K, Cowburn RF, Backstrom A, Vasange M (2004) The retinoic acid and brain-derived neurotrophic factor differentiated SH-SY5Y cell line as a model for Alzheimer's diseaselike tau phosphorylation. Biochem Biophys Res Commun 319:993-1000. doi:10.1016/j.bbrc.2004.05.075
Kamentsky L et al (2011) Improved structure, function and compatibility for Cell Profiler: modular high-throughput image analysis software. Bioinformatics 27:1179-1180. doi:10.1093/bioinfor matics/btr095

Kaplan DR, Matsumoto K, Lucarelli E, Thiele CJ (1993) Induction of TrkB by retinoic acid mediates biologic responsiveness to BDNF and differentiation of human neuroblastoma cells. Eukaryotic Signal Transduction Group. Neuron 11:321-331

Ke YD, Dramiga J, Schutz U, Kril JJ, Ittner LM, Schroder H, Gotz J (2012) Tau-mediated nuclear depletion and cytoplasmic accumulation of SFPQ in Alzheimer's and Pick's disease. PLoS One 7:e35678. doi:10.1371/journal.pone.0035678

Korecka JA et al (2013) Phenotypic characterization of retinoic acid differentiated SH-SY5Y cells by transcriptional profiling. PLoS One 8:e63862. doi:10.1371/journal.pone.0063862

Kou W, Luchtman D, Song C (2008) Eicosapentaenoic acid (EPA) increases cell viability and expression of neurotrophin receptors in retinoic acid and brain-derived neurotrophic factor differentiated SH-SY5Y cells. Eur J Nutr 47:104-113. doi:10.1007/ s00394-008-0703-1

Koussounadis A, Langdon SP, Um IH, Harrison DJ, Smith VA (2015) Relationship between differentially expressed mRNA and mRNA-protein correlations in a xenograft model system. Sci Rep 5:10775. doi:10.1038/Srep10775

Kovalevich J, Langford D (2013) Considerations for the use of SHSY5Y neuroblastoma cells in neurobiology. Methods Mol Biol 1078:9-21. doi:10.1007/978-1-62703-640-5_2

Krishna A et al (2014) Systems genomics evaluation of the SH-SY5Y neuroblastoma cell line as a model for Parkinson's disease. BMC Genom 15:1154. doi:10.1186/1471-2164-15-1154

Kume T et al (2008) Dibutyryl cyclic AMP induces differentiation of human neuroblastoma SH-SY5Y cells into a noradrenergic phenotype. Neurosci Lett 443:199-203. doi:10.1016/j.neulet. 2008.07.079

Lim J, Choi HS, Choi HJ (2015) Estrogen-related receptor gamma regulates dopaminergic neuronal phenotype by activating GSK3beta/NFAT signaling in SH-SY5Y cells. J Neurochem 133:544-557. doi:10.1111/jnc. 13085

Lopes FM et al (2010) Comparison between proliferative and neuronlike SH-SY5Y cells as an in vitro model for Parkinson disease studies. Brain Res 1337:85-94. doi:10.1016/j.brainres.2010.03. 102

Lopes FM et al (2012) Evaluation of the neurotoxic/neuroprotective role of organoselenides using differentiated human neuroblastoma SH-SY5Y cell line challenged with 6-hydroxydopamine. Neurotox Res 22:138-149. doi:10.1007/s12640-012-9311-1

Lopez-Carballo G, Moreno L, Masia S, Perez P, Barettino D (2002) Activation of the phosphatidylinositol 3-kinase/Akt signaling pathway by retinoic acid is required for neural differentiation of SH-SY5Y human neuroblastoma cells. J Biol Chem 277: 25297-25304. doi:10.1074/jbc.M201869200

Mann F, Rougon G (2007) Mechanisms of axon guidance: membrane dynamics and axonal transport in semaphorin signalling. J Neurochem 102:316-323. doi:10.1111/j.1471-4159.2007.04578.x

Meichsner M, Doll T, Reddy D, Weisshaar B, Matus A (1993) The low molecular weight form of microtubule-associated protein 2 is transported into both axons and dendrites. Neuroscience $54: 873-880$

Meijering E (2010) Neuron tracing in perspective. Cytometry A 77:693-704. doi:10.1002/cyto.a.20895

Middlemas DS, Kihl BK, Zhou J, Zhu X (1999) Brain-derived neurotrophic factor promotes survival and chemoprotection of human neuroblastoma cells. J Biol Chem 274:16451-16460

Mlechkovich G et al (2014) Distinct cytoplasmic domains in PlexinA4 mediate diverse responses to semaphorin $3 \mathrm{~A}$ in developing 
mammalian neurons. Sci Signal 7:ra24. doi:10.1126/scisignal. 2004734

Myers JP, Santiago-Medina M, Gomez TM (2011) Regulation of axonal outgrowth and pathfinding by integrin-ECM interactions. Dev Neurobiol 71:901-923. doi:10.1002/dneu.20931

Nakamura M, Matsuo T, Stauffer J, Neckers L, Thiele CJ (2003) Retinoic acid decreases targeting of p27 for degradation via an $\mathrm{N}$-myc-dependent decrease in p27 phosphorylation and an N-myc-independent decrease in Skp2. Cell Death Differ 10:230-239. doi:10.1038/sj.cdd.4401125

Pahlman S, Ruusala AI, Abrahamsson L, Mattsson ME, Esscher T (1984) Retinoic acid-induced differentiation of cultured human neuroblastoma cells: a comparison with phorbolester-induced differentiation. Cell Differ 14:135-144

Palomo GM, Cerrato T, Gargini R, Diaz-Nido J (2011) Silencing of frataxin gene expression triggers p53-dependent apoptosis in human neuron-like cells. Hum Mol Genet 20:2807-2822. doi:10. 1093/hmg/ddr187

Ross RA, Spengler BA (2007) Human neuroblastoma stem cells. Semin Cancer Biol 17:241-247. doi:10.1016/j.semcancer.2006. 04.006

Ross RA, Spengler BA, Biedler JL (1983) Coordinate morphological and biochemical interconversion of human neuroblastoma cells. J Natl Cancer Inst 71:741-747

Sarkanen JR, Nykky J, Siikanen J, Selinummi J, Ylikomi T, Jalonen TO (2007) Cholesterol supports the retinoic acid-induced synaptic vesicle formation in differentiating human SH-SY5Y neuroblastoma cells. J Neurochem 102:1941-1952. doi:10.1111/ j.1471-4159.2007.04676.x

Schneider L et al (2011) Differentiation of SH-SY5Y cells to a neuronal phenotype changes cellular bioenergetics and the response to oxidative stress. Free Radic Biol Med 51:2007-2017. doi:10.1016/ j.freeradbiomed.2011.08.030

Schneider CA, Rasband WS, Eliceiri KW (2012) NIH Image to ImageJ: 25 years of image analysis. Nat Methods 9:671-675

Scifo E et al (2013) Drafting the CLN3 protein interactome in SHSY5Y human neuroblastoma cells: a label-free quantitative proteomics approach. J Proteome Res 12:2101-2115. doi:10. $1021 / \mathrm{pr} 301125 \mathrm{k}$

Scifo E et al (2015) Proteomic analysis of the palmitoyl protein thioesterase 1 interactome in SH-SY5Y human neuroblastoma cells. J Proteom 123:42-53. doi:10.1016/j.jprot.2015.03.038
Shelly M, Cancedda L, Lim BK, Popescu AT, Cheng PL, Gao H, Poo MM (2011) Semaphorin3A regulates neuronal polarization by suppressing axon formation and promoting dendrite growth. Neuron 71:433-446. doi:10.1016/j.neuron.2011.06.041

Shiohira H, Kitaoka A, Enjoji M, Uno T, Nakashima M (2012) Am 80 induces neuronal differentiation via increased tropomyosinrelated kinase B expression in a human neuroblastoma $\mathrm{SH}-$ SY5Y cell line. Biomed Res 33:291-297

Song M et al (2015) Slitrk5 mediates BDNF-dependent TrkB receptor trafficking and signaling. Dev Cell 33:690-702. doi:10.1016/j. devcel.2015.04.009

Thiede-Stan NK, Schwab ME (2015) Attractive and repulsive factors act through multi-subunit receptor complexes to regulate nerve fiber growth. J Cell Sci 128:2403-2414. doi:10.1242/jcs.165555

Tran TS, Kolodkin AL, Bharadwaj R (2007) Semaphorin regulation of cellular morphology. Annu Rev Cell Dev Biol 23:263-292. doi:10.1146/annurev.cellbio.22.010605.093554

Vitriol EA, Zheng JQ (2012) Growth cone travel in space and time: the cellular ensemble of cytoskeleton, adhesion, and membrane. Neuron 73:1068-1081. doi:10.1016/j.neuron.2012.03.005

Vogel C, Marcotte EM (2012) Insights into the regulation of protein abundance from proteomic and transcriptomic analyses. Nat Rev Genet 13:227-232. doi:10.1038/nrg3185

Walton JD et al (2004) Characteristics of stem cells from human neuroblastoma cell lines and in tumors. Neoplasia 6:838-845. doi:10.1593/neo.04310

Wang Z, Gerstein M, Snyder M (2009) RNA-Seq: a revolutionary tool for transcriptomics. Nat Rev Genet 10:57-63. doi:10.1038/ $\operatorname{nrg} 2484$

Wiese C et al (2004) Nestin expression-a property of multi-lineage progenitor cells? Cell Mol Life Sci 61:2510-2522. doi:10.1007/ s00018-004-4144-6

Yaron A, Huang PH, Cheng HJ, Tessier-Lavigne M (2005) Differential requirement for Plexin-A3 and -A4 in mediating responses of sensory and sympathetic neurons to distinct class 3 semaphorins. Neuron 45:513-523. doi:10.1016/j.neuron.2005. 01.013

Zhou Y, Gunput RA, Pasterkamp RJ (2008) Semaphorin signaling: progress made and promises ahead. Trends Biochem Sci 33:161-170. doi:10.1016/j.tibs.2008.01.006 